TRANSACTIONS OF THE

AMERICAN MATHEMATICAL SOCIETY

Volume 361, Number 1, January 2009, Pages 125-160

S 0002-9947(08)04610-2

Article electronically published on August 13, 2008

\title{
THE REGULARITY AND NEUMANN PROBLEM FOR NON-SYMMETRIC ELLIPTIC OPERATORS
}

\author{
CARLOS E. KENIG AND DAVID J. RULE
}

\begin{abstract}
We establish optimal $L^{p}$ bounds for the non-tangential maximal function of the gradient of the solution to a second-order elliptic operator in divergence form, possibly non-symmetric, with bounded measurable coefficients independent of the vertical variable, on the domain above a Lipschitz graph in the plane, in terms of the $L^{p}$-norm at the boundary of the tangential derivative of the Dirichlet data, or of the Neumann data.
\end{abstract}

\section{INTRODUCTION}

We will consider the Dirichlet problem

$$
\begin{cases}L u=0, & \text { in } \Omega \\ u=f_{0}, & \text { on } \partial \Omega\end{cases}
$$

with boundary data $f_{0}$ and the Neumann problem

$$
\begin{cases}L u=0, & \text { in } \Omega \\ \nu \cdot A \nabla u=g_{0}, & \text { on } \partial \Omega\end{cases}
$$

with boundary data $g_{0}$. Here $\nu$ is the outward unit normal vector to $\partial \Omega$ and $L=\operatorname{div} A \nabla$. is an elliptic operator in divergence form with coefficient matrix $A=\left(a_{i j}\right)_{i j}$. The matrix $A$ is assumed to have real-valued bounded measurable entries $\left(\max _{i, j}\left\|a_{i j}\right\|_{L^{\infty}(\Omega)}=\Lambda<\infty\right)$ and satisfy the uniform ellipticity condition

$$
\lambda|\xi|^{2} \leq \xi \cdot A \xi
$$

for some $\lambda>0$ and all $\xi \in \mathbf{R}^{2}$, but $A$ is not necessarily symmetric. The domain $\Omega=\left\{X=(x, t) \in \mathbf{R}^{2} \mid \phi(x)<t\right\}$ is the domain above the graph of a Lipschitz function $\phi$. In the sequel we will denote by $\tau$ the tangent $\left(1, \phi^{\prime}\right) /\left(1+\left(\phi^{\prime}\right)^{2}\right)^{\frac{1}{2}}$ to $\partial \Omega$ and $\partial_{\tau}=\tau \cdot \nabla$ the derivative along the boundary. The conormal derivative will be $\nu \cdot A \nabla$.

Let us begin by fixing some notation. The spaces $C^{\infty}$ and $C_{0}^{\infty}$ are the spaces of smooth functions and smooth functions with compact support, respectively. For clarity, we will often indicate the domain of such functions, for example $C_{0}^{\infty}(\mathbf{R})$. For $p \in[1, \infty]$ and $E$ a subset of either $\mathbf{R}$ or $\mathbf{R}^{2}$, the space $L^{p}(E)$ is the set of measurable functions $f: E \rightarrow \mathbf{R}$ such that $\|f\|_{L^{p}(E)}$ is finite, where

$$
\|f\|_{L^{p}(E)}= \begin{cases}\left(\int_{E}|f|^{p}\right)^{\frac{1}{p}}, & \text { if } p \in[1, \infty) \\ {\operatorname{ess} \sup _{E}|f|,} \text { if } p=\infty\end{cases}
$$

Received by the editors October 24, 2006.

2000 Mathematics Subject Classification. Primary 35J25; Secondary 31A25.

The first author was supported in part by NSF grant number DMS-0456583.

(C)2008 American Mathematical Society 125

Reverts to public domain 28 years from publication 
is defined with respect to the appropriate Lebesgue measure. We define $L^{p}(E, \mathcal{M})$ similarly for functions $F$ with values in $\mathcal{M}$, the set of real-valued $2 \times 2$ matrices, by replacing the absolute value with $|F|:=\sup _{i j}\left|f_{i j}\right|$ where $F=\left(f_{i j}\right)_{i j}$. The set $W^{1, p}(E)$ is the familiar Sobolev space, consisting of functions in $L^{p}(E)$ whose firstorder derivatives (in the sense of distributions) also belong to $L^{p}(E)$ and $W_{\text {loc }}^{1, p}(E)$, the set consisting of those functions in $W^{1, p}\left(E^{\prime}\right)$ for every compact subset $E^{\prime}$ of $E$. We will consider the non-tangential approach regions

$$
\Gamma(Q)=\{X \in \Omega|| X-Q \mid \leq(1+a) \operatorname{dist}(X, \partial \Omega)\}
$$

for each $Q \in \partial \Omega$ ( $a>0$ fixed). Here $\operatorname{dist}(X, \partial \Omega)=\inf _{Q \in \partial \Omega}|Q-X|$. Recall the non-tangential maximal function for a function $u$ on $\Omega$ is a function $N(u): \partial \Omega \rightarrow \mathbf{R}$ given by

$$
N(u)(Q)=\sup _{\Gamma(Q)}|u|
$$

and the related version

$$
\widetilde{N}(u)(Q)=\sup _{X \in \Gamma(Q)}\left(\frac{1}{\left|B_{\delta(X) / 2}(X)\right|} \int_{B_{\delta(X) / 2}(X)}|u|^{2}\right)^{\frac{1}{2}} .
$$

Here $|E|$ is the Lebesgue measure of a set $E$ and $B_{r}=B_{r}(X)=\{Y|| X-Y \mid<r\}$ is the ball centred at $X$ of radius $r$. The notation $\delta(X)$ is an abbreviation for $\operatorname{dist}(X, \partial \Omega)$.

To solve (1.1) and (1.2) we will use the following weak formulations. It will be useful to introduce the space $\widetilde{W}^{1,2}(\Omega)$ of functions $f$ for which the norm

$$
\|f\|_{\widetilde{W}^{1,2}(\Omega)}:=\left(\int_{\Omega}|f(X)|^{2} \frac{d X}{\left(1+|X|^{2}\right)}+\int_{\Omega}|\nabla f(X)|^{2} d X\right)^{\frac{1}{2}}
$$

is finite and the space $\widetilde{L}^{2}(\partial \Omega)$ of functions $f$ for which the norm

$$
\|f\|_{\widetilde{L}^{2}(\partial \Omega)}:=\left(\int_{\partial \Omega}|f(X)|^{2} \frac{d \sigma(X)}{(1+|X|)}\right)^{\frac{1}{2}}
$$

is finite, where $d \sigma$ is the Lebesgue surface measure on $\partial \Omega$. Denote by $\operatorname{Tr}: \widetilde{W}^{1,2}(\Omega) \rightarrow$ $\widetilde{L}^{2}(\partial \Omega)$ the trace operator initially defined on smooth functions $\varphi$ as $\operatorname{Tr}(\varphi)=\left.\varphi\right|_{\partial \Omega}$ and by $\widetilde{W}_{0}^{1,2}(\Omega)$ the set of all $f \in \widetilde{W}^{1,2}(\Omega)$ with $\operatorname{Tr}(f)=0$. (One may readily check that $\operatorname{Tr}$ has a continuous extension to $\widetilde{W}^{1,2}(\Omega)$, just as one shows $\operatorname{Tr}: W^{1,2}(\Omega) \rightarrow$ $L^{2}(\partial \Omega)$ is bounded (see, for example, [11, p. 258]).)

Lemma 1.1. Given $f_{0} \in W^{1,2}(\partial \Omega) \cap L^{\frac{7}{6}}(\partial \Omega) \cap L^{\frac{17}{6}}(\partial \Omega)$, there exists a unique $u \in \widetilde{W}^{1,2}(\Omega)$ such that $\operatorname{Tr}(u)=f_{0}$ and

$$
\int_{\Omega} A \nabla u \cdot \nabla \varphi=0
$$

for all $\varphi \in \widetilde{W}_{0}^{1,2}(\Omega)$. Moreover, there exists a constant $C$, depending only on $\lambda, \Lambda$ and $\Omega$, such that

$$
\|u\|_{\widetilde{W}^{1,2}(\Omega)} \leq C\left(\left\|f_{0}\right\|_{W^{1,2}(\partial \Omega)}+\left\|f_{0}\right\|_{L^{7 / 6}(\partial \Omega)}+\left\|f_{0}\right\|_{L^{17 / 6}(\partial \Omega)}\right) .
$$


We call the $u$ from Lemma 1.1 the solution to (1.1) with data $f_{0}$. Let $H^{1}(\partial \Omega)$ denote the classical Hardy space $H^{1}(\mathbf{R})$ (see, for example, [24, p. 87]) projected onto $\partial \Omega$, that is,

$$
H^{1}(\partial \Omega)=\left\{f: \partial \Omega \rightarrow \mathbf{R} \mid x \mapsto f(x, \phi(x))\left(1+\phi^{\prime}(x)^{2}\right)^{\frac{1}{2}} \in H^{1}(\mathbf{R})\right\}
$$

equipped with the obvious norm. We also introduce the homogeneous Sobolev space

$$
\dot{W}^{1,2}(\Omega)=\left\{u: \Omega \rightarrow \mathbf{R} \mid\|\nabla u\|_{L^{2}(\Omega)}<\infty\right\},
$$

with norm $\|\cdot\|_{\dot{W}^{1,2}(\Omega)}=\|\nabla \cdot\|_{L^{2}(\Omega)}$.

Lemma 1.2. Given $g_{0} \in H^{1}(\partial \Omega)$, there exists a unique $u \in \dot{W}^{1,2}(\Omega)$ such that

$$
\int_{\Omega} A \nabla u \cdot \nabla \varphi=\int_{\partial \Omega} g_{0} \operatorname{Tr}(\varphi) d \sigma
$$

for all $\varphi \in \dot{W}^{1,2}(\Omega)$. Moreover, there exists a constant $C$, depending only on $\lambda, \Lambda$ and $\Omega$, such that

$$
\|u\|_{\dot{W}^{1,2}(\Omega)} \leq C\left\|g_{0}\right\|_{H^{1}(\partial \Omega)} .
$$

We call the $u$ from Lemma 1.2 the solution to (1.2) with data $g_{0}$. We will postpone the proofs of these lemmata until Section 2 Since, in general, we cannot assign a meaning to $\left.\nu \cdot A \nabla u\right|_{\partial \Omega}$, we should be careful as to exactly how we interpret the statement in (1.2) that $\nu \cdot A \nabla u=g_{0}$ on $\partial \Omega$. It is well known that existence of the estimates in the following definition enables a certain non-tangential convergence to the boundary data to be established (see, for example, [21]).

Definition 1.3. (i) We say that the Dirichlet problem holds for $p$, or $(D)_{p}^{A}=(D)_{p}$ holds, if for any $u$ solving (1.1) with boundary data $f_{0} \in L^{p}(\partial \Omega) \cap W^{1,2}(\partial \Omega) \cap$ $L^{\frac{7}{6}}(\partial \Omega) \cap L^{\frac{17}{6}}(\partial \Omega)$ we have

$$
\|N(u)\|_{L^{p}(\partial \Omega)} \leq C(p)\left\|f_{0}\right\|_{L^{p}(\partial \Omega)} .
$$

(ii) We say that the Neumann problem holds for $p$, or $(N)_{p}^{A}=(N)_{p}$ holds, if for any $u$ solving (1.2) with boundary data $g_{0} \in L^{p}(\partial \Omega) \cap H^{1}(\partial \Omega)$ we have

$$
\|\tilde{N}(\nabla u)\|_{L^{p}(\partial \Omega)} \leq C(p)\left\|g_{0}\right\|_{L^{p}(\partial \Omega)} .
$$

(iii) We say that the regularity problem holds for $p$, or $(R)_{p}^{A}=(R)_{p}$ holds, if for any $u$ solving (1.1) with boundary data $f_{0} \in W^{1, p}(\partial \Omega) \cap W^{1,2}(\partial \Omega) \cap L^{\frac{7}{6}}(\partial \Omega) \cap L^{\frac{17}{6}}(\partial \Omega)$ we have

$$
\|\tilde{N}(\nabla u)\|_{L^{p}(\partial \Omega)} \leq C(p)\left\|\partial_{\tau} f_{0}\right\|_{L^{p}(\partial \Omega)} .
$$

In each case, the constant $C(p)>0$ must depend only on $\lambda, \Lambda, \Omega$ and $p$.

The main result of this paper is the following.

Theorem 1.4. Let $L=\operatorname{div} A \nabla$ be an elliptic operator as defined above with coefficient matrix $A=A(x)$ independent of the $t$-variable in the domain $\Omega=\{X=$ $\left.(x, t) \in \mathbf{R}^{2} \mid \phi(x)<t\right\}$ above the graph of a Lipschitz function $\phi$. Then $(N)_{p}$ and $(R)_{p}$ hold for some (possibly small) $p>1$.

In 2000, Kenig, Koch, Pipher and Toro [19] showed that the Dirichlet problem for an elliptic operator $L=\operatorname{div} A \nabla$ in $\Omega \subset \mathbf{R}^{2}$ holds for some $p \geq 2$, where $A$ has coefficients independent of the $t$-direction. The advance here was that the $2 \times 2$ matrix $A$ did not need to be assumed symmetric. The exponent $p$ could not in 
general be specified, as they showed with an example. This example can be used to show the same is true in our case: For any given $p$ there exist operators $L$ as in Theorem 1.4 for which $(N)_{p}$ and $(R)_{p}$ do not hold. (This example is worked out in the Appendix.) Jerison and Kenig showed in [16] that when $A$ is also symmetric, $(D)_{2}$ holds. In 21], Kenig and Pipher show that if $(R)_{p}^{A}$ holds in $\Omega$ for some $p$, then $(D)_{p^{\prime}}^{A^{t}}$ holds, where $\frac{1}{p}+\frac{1}{p^{\prime}}=1$ and $A^{t}$ is the transpose of $A$. Here we will prove Theorem 1.4 by showing a reverse implication: if $(D)_{p^{\prime}}^{A^{t}}$ holds and $A$ is independent of the $t$-direction, then $(N)_{p}^{\widetilde{A}}$ and $(R)_{p}^{A}$ hold, where $\widetilde{A}=A^{t} / \operatorname{det}(A)$. Then, as a simple corollary, the main result in [19] passes over to the Neumann and regularity problems to give Theorem 1.4.

The layout of this paper is as follows. In Section 2 we fix some more notation and lay out some preliminaries. In Section 3 we begin by observing that a change of variables used in [19] can reduce our problem to coefficient matrices $A$ which are upper triangular. We then go on to show that the solvability of the Dirichlet problem allows us to reduce Theorem 1.4 to the boundedness of certain layer potentials. We remark that Verchota [25] reduced similar regularity results for the Laplacian to proving the invertibility of the classical layer potentials. In Section 4 , this boundedness is proved in the special case that the boundary of our domain has a small Lipschitz constant. In Section 5 we use David's method [9] to show that the layer potentials must also be bounded on domains $\Omega$ when $\phi$ is an arbitrary Lipschitz function. As an appendix, we show the example in [19] serves the same purpose for Theorem 1.4 as it does for their Theorem 3.1.

As is common practice the letter $C$ will denote a constant whose value may change from line to line, but which can be fixed depending only on the constants $\lambda, \Lambda$ and the Lipschitz constant of $\phi$. When a subscript is added the value will not vary from line to line, and when the constant depends on other parameters or when we wish to record the form of the dependency this will be noted explicity (for example, we wrote above $C=C(p))$.

\section{Preliminaries}

The square function $S(f): \partial \Omega \rightarrow \mathbf{R}$ of $f: \Omega \rightarrow \mathbf{R}$ is given by

$$
S(f)(Q)=\left\{\int_{\Gamma(Q)}|\nabla f|^{2}\right\}^{\frac{1}{2}}
$$

and the Hardy-Littlewood maximal operator $M: L^{p}(\partial \Omega) \rightarrow L^{p}(\partial \Omega)(1<p \leq \infty)$ is given by

$$
M(f)(Q)=\sup _{r>0} \frac{1}{\sigma\left(\Delta_{r}(Q)\right)} \int_{\Delta_{r}(Q)}|f| d \sigma,
$$

where $\Delta_{r}(Q)=B_{r}(Q) \cap \partial \Omega$ and $d \sigma$ is the Lebesgue surface measure of $\partial \Omega$. We also define $M$ acting on functions $F: \mathbf{R} \rightarrow \mathcal{M}$ analogously, replacing the absolute value with the corresponding matrix version and taking the supremum over intervals in R. A function $K: \mathbf{R}^{2} \rightarrow \mathcal{M}$ is said to be a Calderón-Zygmund kernel if there exist constants $C>0$ and $\alpha \in(0,1]$ such that

$$
|K(x, y)| \leq \frac{C}{|x-y|}
$$


for all $x, y \in \mathbf{R}, x \neq y$,

$$
\left|K(x, y)-K\left(x^{\prime}, y\right)\right| \leq \frac{C\left|x-x^{\prime}\right|^{\alpha}}{\left(|x-y|+\left|x^{\prime}-y\right|\right)^{1+\alpha}}
$$

when $\left|x-x^{\prime}\right| \leq \frac{1}{2} \max \left\{|x-y|,\left|x^{\prime}-y\right|\right\}$, and

$$
\left|K(x, y)-K\left(x, y^{\prime}\right)\right| \leq \frac{C\left|y-y^{\prime}\right|^{\alpha}}{\left(|x-y|+\left|x-y^{\prime}\right|\right)^{1+\alpha}}
$$

when $\left|y-y^{\prime}\right| \leq \frac{1}{2} \max \left\{|x-y|,\left|x-y^{\prime}\right|\right\}$. Recall, for $F=\left(f_{i j}\right)_{i j},|F|:=\sup _{i j}\left|f_{i j}\right|$. For Banach spaces $\mathcal{X}$ and $\mathcal{Y}$ dense in $L^{2}(\mathbf{R}, \mathcal{M})$, a continuous linear operator $T: \mathcal{X} \rightarrow \mathcal{Y}^{\prime}$ is said to be a singular integral operator associated to the CalderónZygmund kernel $K$ if for each $F \in \mathcal{X}$ and $G \in \mathcal{Y}$ with disjoint support, we have the representation

$$
\langle G, T(F)\rangle=\int_{\mathbf{R}^{2}} G(x)^{t} K(x, y) F(y) d y d x,
$$

where $\langle\cdot, \cdot\rangle$ is the standard inner product on $L^{2}(\mathbf{R}, \mathcal{M})$ and $G(x)^{t}$ is the transpose of $G(x)$. The maximal singular integral operator $T^{*}$ associated to $K$ is defined by first setting

$$
T^{(\delta)}(F)(x)=\int_{|y-x| \geq \delta} K(x, y) F(y) d y
$$

and then $T^{*}(F)=\sup _{\delta>0}\left|T^{(\delta)}(F)\right|$. The following lemmata are well known for any elliptic operator as defined above (see, for example, [18] and [13, Thm. 8.24]).

Lemma 2.1. Let $u \in H^{1,2}\left(B_{2 r}\right)$ satisfy Lu $=0$. Then there exists a constant $C>0$ which depends only on $\lambda$ and $\Lambda$ such that

$$
f_{B_{r}}|\nabla u(Z)|^{2} d Z \leq \frac{C}{r^{2}} f_{B_{2 r}}|u(Z)|^{2} d Z .
$$

Lemma 2.2. For $u \in H^{1,2}\left(B_{5 r / 4}\right)$ such that $L u=0$ and $p \geq 1$, there exists a constant $C(p)>0$ which depends only on $\lambda, \Lambda$ and $p$ such that

$$
\sup _{B_{r / 2}}|u| \leq C(p)\left(f_{B_{r}}|u|^{p}\right)^{\frac{1}{p}} .
$$

Lemma 2.3. For $u \in H^{1,2}\left(B_{3 r / 2}\right)$ such that $L u=0$, there exist constants $C>0$ and $p>2$ which depend only on $\lambda$ and $\Lambda$ such that

$$
\left(f_{B_{r / 2}}|\nabla u|^{p}\right)^{\frac{1}{p}} \leq C\left(f_{B_{r}}|\nabla u|^{2}\right)^{\frac{1}{2}} .
$$

Lemma 2.4. Let $u \in H^{1,2}\left(B_{r}\right)$ satisfy $L u=0$. Then there exist constants $C>0$ and $\alpha \in(0,1]$, depending only on $\lambda$ and $\Lambda$, such that

$$
|u(X)-u(Y)| \leq \frac{C}{r^{1+\alpha}}\|u\|_{L^{2}\left(B_{r}\right)}|X-Y|^{\alpha},
$$

for all $X, Y \in B_{2 r / 3}$.

We will now prove Lemmata 1.1 and 1.2 . 
Proof of Lemma 1.1. Without loss of generality we suppose $\Omega=\mathbf{R}_{+}^{2}=\{(x, t) \mid t>$ $0\}$, since the transformation $\Phi: \Omega \rightarrow \mathbf{R}_{+}^{2}$ given by $\Phi(x, t)=(x, t-\phi(x))$ will yield the general case. Let $P_{t}: \mathbf{R} \rightarrow \mathbf{R}$ denote the Poisson kernel:

$$
P_{t}(x):=\frac{t}{\pi\left(|x|^{2}+t^{2}\right)} \text {. }
$$

Let

$$
w(x, t)=f_{0} * P_{t}(x):=\int_{\mathbf{R}} f_{0}(x-y) P_{t}(y) d y
$$

be the harmonic extension of $f_{0}$ to $\mathbf{R}_{+}^{2}$. We will show that

$$
\|w\|_{\widetilde{W}^{1,2}\left(\mathbf{R}_{+}^{2}\right)} \leq C\left(\left\|f_{0}\right\|_{W^{1,2}\left(\partial \mathbf{R}_{+}^{2}\right)}+\left\|f_{0}\right\|_{L^{7 / 6}\left(\partial \mathbf{R}_{+}^{2}\right)}+\left\|f_{0}\right\|_{L^{17 / 6}\left(\partial \mathbf{R}_{+}^{2}\right)}\right) .
$$

Using Hölder's inequality,

$$
\int_{\mathbf{R}_{+}^{2}}|w(X)|^{2} \frac{d X}{\left(1+|X|^{2}\right)} \leq C\|w\|_{L^{17 / 6}\left(\mathbf{R}_{+}^{2}\right)}^{2}
$$

but, by Fubini's Theorem,

$$
\begin{aligned}
\|w\|_{L^{17 / 6}\left(\mathbf{R}_{+}^{2}\right)}^{\frac{17}{6}} & =\int_{0}^{\infty}\left\|f_{0} * P_{t}\right\|_{L^{17 / 6}(\mathbf{R})}^{\frac{17}{6}} d t \\
& =\int_{0}^{1}\left\|f_{0} * P_{t}\right\|_{L^{17 / 6}(\mathbf{R})}^{\frac{17}{6}} d t+\int_{1}^{\infty}\left\|f_{0} * P_{t}\right\|_{L^{17 / 6}(\mathbf{R})}^{\frac{17}{6}} d t .
\end{aligned}
$$

Now, using well-known properties of the Poisson kernel and Young's inequality (see, for example, 23]), we can bound this by

$$
\begin{aligned}
& \left\|M\left(f_{0}\right)\right\|_{L^{17 / 6}(\mathbf{R})}^{\frac{17}{6}}+\left\|f_{0}\right\|_{L^{7 / 6}(\mathbf{R})}^{\frac{17}{6}} \int_{1}^{\infty}\left\|P_{t}\right\|_{L^{119 / 59}(\mathbf{R})}^{\frac{17}{6}} d t \\
& \leq C\left\|f_{0}\right\|_{L^{17 / 6}(\mathbf{R})}^{\frac{17}{6}}+C\left\|f_{0}\right\|_{L^{7 / 6}(\mathbf{R})}^{\frac{17}{6}},
\end{aligned}
$$

since $\left\|P_{t}\right\|_{L^{119 / 59}(\mathbf{R})}^{\frac{17}{6}}=C t^{-\frac{10}{7}}$. This controls the first integral in the $\widetilde{W}^{1,2}\left(\mathbf{R}_{+}^{2}\right)$-norm of $w$. To control the second, first observe that

$$
\left\|P_{t}^{\prime}\right\|_{L^{1}(\mathbf{R})}=C t^{-2}
$$

so, as before,

$$
\begin{aligned}
& \left\|\partial_{1} w\right\|_{L^{2}\left(\mathbf{R}_{+}^{2}\right)} \leq \int_{0}^{1}\left\|f_{0}^{\prime} * P_{t}\right\|_{L^{2}(\mathbf{R})} d t+\int_{1}^{\infty}\left\|f_{0} * P_{t}^{\prime}\right\|_{L^{2}(\mathbf{R})} d t \\
& \leq\left\|M\left(f_{0}^{\prime}\right)\right\|_{L^{2}(\mathbf{R})}+\left\|f_{0}\right\|_{L^{2}(\mathbf{R})} \int_{1}^{\infty}\left\|P_{t}^{\prime}\right\|_{L^{1}(\mathbf{R})} d t \leq C\left\|f_{0}\right\|_{H^{1,2}\left(\partial \mathbf{R}_{+}^{2}\right)} .
\end{aligned}
$$

We may control $\left\|\partial_{2} w\right\|_{L^{2}\left(\mathbf{R}_{+}^{2}\right)}$ similarly since (via the Cauchy-Riemann equations) we know $\partial_{2} w=-\partial_{1} \widetilde{w}$, where $\widetilde{w}(x, t)=H\left(f_{0}\right) * P_{t}(x)$ and $H: L^{2}\left(\partial \mathbf{R}_{+}^{2}\right) \rightarrow L^{2}\left(\partial \mathbf{R}_{+}^{2}\right)$ is the Hilbert transform. Thus (2.5) is proved.

Now we will show

$$
(\psi, \varphi) \mapsto \int_{\mathbf{R}_{+}^{2}} A \nabla \psi \cdot \nabla \varphi
$$

defines a bounded coercive bilinear form on $\widetilde{W}_{0}^{1,2}\left(\mathbf{R}_{+}^{2}\right)$. Linearity and boundedness are clear, so to prove coercivity we first observe that $\psi \in \widetilde{W}_{0}^{1,2}\left(\mathbf{R}_{+}^{2}\right)$ can be extended to a function in $\widetilde{W}^{1,2}\left(\mathbf{R}^{2}\right)$ (the space with norm (1.4) but integrating 
over $\mathbf{R}^{2}$ ), which we will also call $\psi$, simply by extending by zero. Then obviously $\|\nabla \psi\|_{L^{2}\left(\mathbf{R}^{2}\right)}=\|\nabla \psi\|_{L^{2}\left(\mathbf{R}_{+}^{2}\right)}$. Thus, using Poincaré's inequality,

$$
\begin{aligned}
\int_{\mathbf{R}^{2}}|\psi(X)|^{2} \frac{d X}{\left(1+|X|^{2}\right)} & \leq C \int_{B_{4}(0)}|\psi|^{2}+C \sum_{n=2}^{\infty} 2^{-2 n} \int_{B_{2^{n}(0) \backslash B_{2^{n-1}}(0)}}|\psi|^{2} \\
& \leq C \int_{B_{4}(0)}|\nabla \psi|^{2}+\left.C \sum_{n=2}^{\infty} \int_{B_{2^{n}(0) \backslash B_{2^{n-1}(0)}}|\nabla \psi|^{2}}\left|\leq \int_{\mathbf{R}^{2}}\right| \nabla \psi\right|^{2}=C \int_{\mathbf{R}_{+}^{2}}|\nabla \psi|^{2} .
\end{aligned}
$$

Combining this with (1.3) easily gives the coercivity.

We may now apply the Lax-Milgram Theorem to find a unique $v \in \widetilde{W}_{0}^{1,2}\left(\mathbf{R}_{+}^{2}\right)$ such that

$$
\int_{\mathbf{R}_{+}^{2}} A \nabla v \cdot \nabla \varphi=\int_{\mathbf{R}_{+}^{2}}-A \nabla w \cdot \nabla \varphi
$$

for all $\varphi \in \widetilde{W}_{0}^{1,2}\left(\mathbf{R}_{+}^{2}\right)$. Then $u=v+w \in \widetilde{W}^{1,2}\left(\mathbf{R}_{+}^{2}\right)$ is clearly the unique function in $\widetilde{W}^{1,2}\left(\mathbf{R}_{+}^{2}\right)$ such that (1.5) holds and $\operatorname{Tr}(u)=f_{0}$. The estimate (1.6) follows from (2.5) and the Lax-Milgram Theorem.

A function $f: \mathbf{R} \rightarrow \mathbf{R}$ is said to be of bounded mean oscillation, written $f \in$ $\mathrm{BMO}$, if

$$
\|f\|_{\mathrm{BMO}}:=\sup _{I} f_{I}\left|f(x)-f_{I} f\right| d x<\infty
$$

where the supremum is taken over all intervals $I \subset \mathbf{R}$.

Proof of Lemma 1.2. Once again, without loss of generality we may assume $\Omega=$ $\mathbf{R}_{+}^{2}$. The bilinear form (2.6) is clearly bounded and coercive on $\dot{W}^{1,2}\left(\mathbf{R}_{+}^{2}\right)$, so to apply the Lax-Milgram Theorem it remains to check that

$$
\varphi \mapsto \int_{\partial \Omega} g_{0} \operatorname{Tr}(\varphi) d \sigma
$$

is a bounded linear functional on $\dot{W}^{1,2}\left(\mathbf{R}_{+}^{2}\right)$ for a given $g_{0} \in H^{1}\left(\partial \mathbf{R}_{+}^{2}\right)$.

It suffices to check $\operatorname{Tr}: \dot{W}^{1,2}\left(\mathbf{R}_{+}^{2}\right) \rightarrow$ BMO is a bounded operator. This is easily done as follows. Let $I=B_{R} \cap \partial \mathbf{R}_{+}^{2}$ and $B_{R}^{+}=B_{R} \cap \mathbf{R}_{+}^{2}$, where $B_{R}$ is a ball with centre on $\partial \mathbf{R}_{+}^{2}$. Denote by $\varphi_{B_{2 R}^{+}}$the average $f_{B_{2 R}^{+}} u$, where $B_{2 R}$ is the ball concentric with $B_{R}$, but with radius $2 R$. Fix $\xi: \overline{\mathbf{R}_{+}^{2}} \rightarrow \mathbf{R}$ to be a smooth cut-off function equal to one near $I$, supported in $B_{2 R}$ and such that $|\nabla \xi| \leq C / R$. Then

$$
\begin{aligned}
f_{I}\left|\varphi-\varphi_{B_{2 R}^{+}}\right| d \sigma & \leq \frac{1}{R} \int_{\partial \mathbf{R}_{+}^{2}} \xi\left|\varphi-\varphi_{B_{2 R}^{+}}\right|=-\frac{1}{R} \int_{B_{2 R}^{+}} \partial_{t}\left(\xi\left|\varphi-\varphi_{B_{2 R}^{+}}\right|\right) \\
& =-\frac{1}{R} \int_{B_{2 R}^{+}}\left(\partial_{t} \xi\right)\left|\varphi-\varphi_{B_{2 R}^{+}}\right|-\frac{1}{R} \int_{B_{2 R}^{+}} \xi\left(\partial_{t} \varphi\right) \operatorname{sgn}\left(\varphi-\varphi_{B_{2 R}^{+}}\right) \\
& \leq \frac{C}{R^{2}} \int_{B_{2 R}^{+}}\left|\varphi-\varphi_{B_{2 R}^{+}}\right|+\frac{1}{R} \int_{B_{2 R}^{+}}|\nabla \varphi| \\
& \leq C\|\nabla \varphi\|_{L^{2}\left(\mathbf{R}_{+}^{2}\right)}=C\|\varphi\|_{\dot{W}^{1,2}\left(\mathbf{R}_{+}^{2}\right)}
\end{aligned}
$$

where the last inequality follows from Hölder's and Poincaré's inequalities. 
Kenig and $\mathrm{Ni}$ [20] provide the following definition and existence of a fundamental solution.

Definition 2.5. A function $\Gamma_{X}: \mathbf{R}^{2} \rightarrow \mathbf{R}$ is called a fundamental solution for $L=\operatorname{div} A \nabla \cdot$ with pole at $X$ if

(i) $\Gamma_{X} \in W_{\text {loc }}^{1,2}\left(\mathbf{R}^{2} \backslash\{X\}\right) \cap W_{\text {loc }}^{1, p}\left(\mathbf{R}^{2}\right)$ for all $p<2$, and, for every $\varphi \in C_{0}^{\infty}\left(\mathbf{R}^{2}\right)$,

$$
\int_{\mathbf{R}^{2}} A^{t}(Y) \nabla \Gamma_{X}(Y) \cdot \nabla \varphi(Y) d Y=-\varphi(X) .
$$

(ii) $\left|\Gamma_{X}(Y)\right|=O(\log |X-Y|)$ as $|Y| \rightarrow \infty$.

Theorem 2.6. For each $X \in \mathbf{R}^{2}$ there exists a unique fundamental solution $\Gamma_{X}$ for $L$ with pole at $X$, and positive constants $C_{1}, C_{2}, R_{1}<1, R_{2}>1$, which depend only on $\lambda$ and $\Lambda$, such that

$$
\begin{aligned}
& C_{1} \log (1 /|X-Y|) \leq-\Gamma_{X}(Y) \leq C_{2} \log (1 /|X-Y|) \quad \text { for } \quad|X-Y|<R_{1}, \quad \text { and } \\
& C_{1} \log (|X-Y|) \leq \Gamma_{X}(Y) \leq C_{2} \log (|X-Y|) \quad \text { for } \quad|X-Y|>R_{2} .
\end{aligned}
$$

The notation we employ to denote differentiation is standard, but let us clarify one point. When the fundamental solution is differentiated, $\nabla \Gamma_{X}(Y)$ will denote the gradient in the $Y$-variable and $\nabla_{X} \Gamma_{X}(Y)$ the gradient in the $X$-variable.

Lemma 2.7. Fix $X, Y \in \mathbf{R}^{2}$. Let $\Gamma_{X}$ be the fundamental solution for an elliptic operator $L$ as defined above with pole at $X$, and $\Gamma_{Y}^{t}$ the fundamental solution to the adjoint operator $L^{t}=\operatorname{div} A^{t} \nabla$ with pole at $Y$. Then we have that

$$
\Gamma_{X}(Y)=\Gamma_{Y}^{t}(X) .
$$

Proof. We will prove the lemma for a modified coefficient matrix $A$ where $A=I$ outside the ball $B_{R}(0)$. It is then easy, using Definition 2.5 and Theorem 2.6, to show that passing to the limit $R \rightarrow \infty$ we obtain the identity for general $A$.

The linear transformation $Z \mapsto C Z(C>0)$, transforms $\Gamma_{X}$ and $\Gamma_{Y}$ into fundamental solutions of operators with the same ellipticity constants $\lambda$ and $\Lambda$. Thus, without loss of generality, we can assume $A=I$ outside $B_{1 / 2}(0)$ and that $X, Y \in B_{1 / 2}(0)$. Green's second identity gives us

$$
\begin{aligned}
\Gamma_{Y}^{t}(X)-\Gamma_{X}(Y)= & \int_{\partial B_{R}(0)} \Gamma_{Y}^{t}(Z) \nu(Z) \cdot A^{t}(Z) \nabla \Gamma_{X}(Z) d \sigma(Z) \\
& -\int_{\partial B_{R}(0)} \Gamma_{X}(Z) \nu(Z) \cdot A(Z) \nabla \Gamma_{Y}^{t}(Z) d \sigma(Z),
\end{aligned}
$$

for any $R>1$. But on the other hand, the uniqueness and the construction used in [20] shows us that, for $Z \in B_{1}(0)^{c}$,

$$
\Gamma_{X}(Z)=g(Z)+w(Z)
$$

and

$$
\Gamma_{Y}^{t}(Z)=h(Z)+v(Z),
$$

where $g$ and $h$ are Green's functions for $B_{1}(X)^{c}$ and $B_{1}(Y)^{c}$, respectively, with poles at infinity, and $w$ and $v$ are harmonic functions satisfying $|w(Z)|+|v(Z)| \leq C|Z|^{-\alpha}$, so then $|\nabla w(Z)|+|\nabla v(Z)| \leq C|Z|^{-(1+\alpha)}$ (see, for example, [13, Thm. 2.10]). Given 
our assumptions on $A$ we have the explicit expressions $g(Z)=\log |Z-X|$ and $h(Z)=\log |Z-Y|$. Thus

$$
\begin{aligned}
\left|\nabla \Gamma_{X}(Z)-\nabla \Gamma_{Y}^{t}(Z)\right| & \leq|\nabla g(Z)-\nabla h(Z)|+|\nabla w(Z)|+|\nabla v(Z)| \\
& \leq C\left(R^{-2}+R^{-(1+\alpha)}\right)
\end{aligned}
$$

and

$$
\left|\Gamma_{X}(Z)-\log \right| Z||+\left|\Gamma_{Y}^{t}(Z)-\log \right| Z|| \leq C\left(R^{-\alpha}+\log |(R+1) / R|\right)
$$

on $\partial B_{R}(0)$. We may conclude, by the above and our assumptions on $A$, that

$$
\begin{aligned}
\left|\Gamma_{Y}^{t}(X)-\Gamma_{X}(Y)\right| & \leq \int_{\partial B_{R}(0)}\left|\left(\Gamma_{Y}^{t}(Z)-(\log |Z|)\right) \nu(Z) \cdot A(Z) \nabla \Gamma_{X}(Z)\right| d \sigma(Z) \\
& +\int_{\partial B_{R}(0)}\left|(\log |Z|) \nu(Z) \cdot\left(A^{t}(Z) \nabla \Gamma_{X}(Z)-A \nabla \Gamma_{Y}^{t}(Z)\right)\right| d \sigma(Z) \\
& +\int_{\partial B_{R}(0)} \mid\left((\log |Z|)-\Gamma_{X}(Z)\right) \nu(Z) \cdot A(Z) \nabla \Gamma_{Y}^{t}(Z) d \sigma(Z) \\
& \leq C\left(R^{-\alpha}+\log |(R+1) / R|+(\log R)\left(R^{-\alpha}+R^{-1}\right)\right),
\end{aligned}
$$

which tends to zero as $R \rightarrow \infty$.

The following theorem was proved by Kenig, Koch, Pipher and Toro 19. They prove that if the $L^{2}$-norms of the square function and non-tangential maximal function of all solutions vanishing at a fixed point can be compared in all bounded Lipschitz domains $\Omega_{0}$ contained in $\Omega$ with constants depending only on the Lipschitz character of the domain, then $(D)_{p}$ holds for some $p$. The estimate

$$
\|S(u)\|_{L^{p}\left(\partial \Omega_{0}\right)} \simeq\|N(u)\|_{L^{p}\left(\partial \Omega_{0}\right)}
$$

for each $p>1$ and solutions $u$ which vanish at a fixed point in $\Omega_{0}$ is demonstrated therein. Dahlberg [5] showed this estimate for $p=2$ implies that solutions are $\varepsilon$ approximable, and in [19, the authors go on to show this $\varepsilon$-approximability implies that $(D)_{p}$ holds for some $p>1$. We refer the reader to [19] for the details and precise definitions, Remark (2.11) providing a brief overview.

Theorem 2.8. Let $L=\operatorname{div} A \nabla$ be an elliptic operator in a domain $\Omega=\{(x, t) \in$ $\left.\mathbf{R}^{2} \mid \phi(x)<t\right\}$, where $A=A(x)$ is independent of the $t$-variable and $\phi: \mathbf{R} \rightarrow \mathbf{R}$ is a Lipschitz function. Then there exists a (possibly large) $p$ such that $(D)_{p}$ holds in $\Omega$, with bound depending only on $\lambda, \Lambda, p$ and the Lipschitz constant of $\phi$.

Given any solution $u$ to an elliptic equation $L u=\operatorname{div} A \nabla u=0$, the vector $A \nabla u$ is divergence free and so can be written as the curl of a vector. This amounts to finding a $\tilde{u}$ solving

$$
\left(\begin{array}{cc}
0 & 1 \\
-1 & 0
\end{array}\right) \nabla \tilde{u}=A \nabla u \text {. }
$$

The function $\tilde{u}$ (defined up to a constant by (2.9)) is called the conjugate of $u$ and $(u, \tilde{u})$ is a conjugate pair $[22$. Observe firstly that $\widetilde{u}$ satisfies an elliptic equation with coefficient matrix $\widetilde{A}=A^{t} / \operatorname{det} A$, and secondly that the conormal derivative of $u$ is the tangential derivative of $\tilde{u}$ and vice versa.

In Sections 3 and 4 we will work under the a priori assumptions that $A=I$ for large $x, A$ and $\phi$ are smooth functions, $\left\|\phi^{\prime}\right\|_{L^{\infty}(\mathbf{R})} \leq k, \phi^{\prime} \equiv \alpha_{0}$ for large $x$ and $x \mapsto \phi(x)-\alpha_{0} x \in C_{0}^{\infty}(\mathbf{R})$. Once our theorems have been proved under our a priori 
assumptions, it is a simple matter to obtain the general case. Note that, under our a priori assumptions, if $u$ solves (1.1) with data $f_{0} \in C_{0}^{\infty}(\partial \Omega)$, then $u \in C^{\infty}(\bar{\Omega})$, and $u(X)=O\left(|X|^{\delta-1}\right)$ and $\nabla u(X)=O\left(|X|^{\delta-2}\right)$ for all $\delta>0$ as $|X| \rightarrow \infty$. Moreover, if

$$
\widetilde{u}(X)=-\int_{\gamma(X)} \nu(Z) \cdot A(Z) \nabla u(Z) d l(Z),
$$

where $\gamma(X)$ is the line segment $\gamma(X)=\{(y, s) \mid s \geq t, y=x, X=(x, t)\}$, $\nu$ is the unit normal to $\gamma(X)$ and $d l$ is arc length, then $(u, \widetilde{u})$ is a conjugate pair and $\widetilde{u}(X)=O\left(|X|^{\delta-1}\right)$ for all $\delta>0$ as $|X| \rightarrow \infty$. (See (4.22) and the lines following it for a similar argument.)

Theorem 2.9. Let $u, \Omega$ and $A$ be as in the previous paragraph. If $p^{\prime}>1$ is such that $(D)_{p^{\prime}}^{A^{t}}$ holds, then there exists a constant $C(p)$, depending only on $\lambda, \Lambda, k, p$ and the $(D)_{p^{\prime}}^{A^{t}}$ constant of $A^{t}$, such that

$$
\|\nabla u\|_{L^{p}(\partial \Omega)} \leq C(p)\left\|\partial_{\tau} f_{0}\right\|_{L^{p}(\partial \Omega)} .
$$

Also, if $u$ solves (1.2) with coefficient matrix $A$ replaced by $\widetilde{A}=A^{t} / \operatorname{det}(A)$ and $u$ verifies the a priori assumptions $u(X)=O\left(|X|^{\delta-1}\right)$ and $\nabla u(X)=O\left(|X|^{\delta-2}\right)$ for all $\delta>0$ as $|X| \rightarrow \infty$, then there exists a constant $C(p)$, depending on the same quantities, such that

$$
\|\nabla u\|_{L^{p}(\partial \Omega)} \leq C(p)\left\|g_{0}\right\|_{L^{p}(\partial \Omega)}
$$

As usual, $\frac{1}{p}+\frac{1}{p^{\prime}}=1$.

We cannot expect these estimates to hold for all $p>1$ as the Appendix shows. The a priori assumptions for solutions to (1.2) will hold if, for instance, $g_{0} \in$ $C_{0}^{\infty}(\partial \Omega) \cap H^{1}(\partial \Omega)$. Such $g_{0}$ are dense in $L^{p}(\partial \Omega), 1<p<\infty$.

Proof of Theorem 2.9. We start out by showing that if $u(X)=O\left(|X|^{\delta-1}\right)$ for all $\delta>0$ as $|X| \rightarrow \infty$, then, for $1<p<\infty$,

$$
\|S(u)\|_{L^{p}(\partial \Omega)} \simeq\|N(u)\|_{L^{p}(\partial \Omega)} .
$$

In fact, let $\Omega_{R}=\Omega \cap B_{R}(0, \phi(0))$, so we have that $A_{R}=(0, R / 2) \in \Omega_{R}$ and $\operatorname{dist}\left(A_{R}, \partial \Omega_{R}\right) \simeq \operatorname{dist}\left(A_{R}, \partial \Omega\right) \simeq R$. By (2.8) we have

$$
\|S(u)\|_{L^{p}\left(\partial \Omega_{R}\right)} \simeq\left\|N\left(u-u\left(A_{R}\right)\right)\right\|_{L^{p}\left(\partial \Omega_{R}\right)},
$$

but since $u(X)=O\left(|X|^{\delta-1}\right),|\nabla u|=O\left(|X|^{\delta-2}\right)$ for all $\delta>0$ we may choose $\delta$ so that $1+(\delta-1) p>0$, so $\left|u\left(A_{r}\right)\right|^{p}\left|\partial \Omega_{R}\right|+\|S(u)\|_{L^{p}\left(\partial \Omega_{R} \backslash \partial \Omega\right)}+\|N(u)\|_{L^{p}\left(\partial \Omega_{R} \backslash \partial \Omega\right)} \rightarrow 0$ as $R \rightarrow \infty$ and (2.13) follows. Now let $f_{0}, u$ and $\widetilde{u}$ be as above but with $A$ replaced by $A^{t}$. Define the operator $H_{A^{t}}$ by $H_{A^{t}}\left(f_{0}\right)=\left.\widetilde{u}\right|_{\partial \Omega}$. This is a bounded operator on $L^{p^{\prime}}(\partial \Omega)$, with norm depending on $\Lambda, p$, the constants in (2.13) and the $(D)_{p^{\prime}}^{A^{t}}$ constant. Indeed,

$$
\begin{aligned}
& \left\|H_{A^{t}}\left(f_{0}\right)\right\|_{L^{p^{\prime}}(\partial \Omega)}=\|\widetilde{u}\|_{L^{p^{\prime}}(\partial \Omega)} \leq\|N(\widetilde{u})\|_{L^{p^{\prime}}(\partial \Omega)} \\
& \simeq\|S(\widetilde{u})\|_{L^{p^{\prime}}(\partial \Omega)} \simeq\|S(u)\|_{L^{p^{\prime}}(\partial \Omega)} \simeq\|N(u)\|_{L^{p^{\prime}}(\partial \Omega)} \leq C(p)\left\|f_{0}\right\|_{L^{p^{\prime}}(\partial \Omega)},
\end{aligned}
$$

Now let us prove the identity

$$
\int_{\partial \Omega}\left(\partial_{\tau}\left(H_{A} g\right)\right) h-g\left(\partial_{\tau}\left(H_{A^{t}} h\right)\right) d \sigma=0
$$


for any $g, h \in C_{0}^{\infty}(\partial \Omega)$. This is easily done as follows: Let $w$ and $v$ be the extensions of $h$ and $g$, respectively, satisfying $\operatorname{div}\left(A^{t} \nabla w\right)=\operatorname{div}(A \nabla v)=0$ in $\Omega$ so that $|w(X)|+|X||\nabla w(X)|+|v(X)|+|X||\nabla v(X)|=O\left(|X|^{\delta-1}\right)$ for large $|X|$. Then

$$
\begin{aligned}
& \int_{\partial \Omega}\left(\partial_{\tau}\left(H_{A} g\right)\right) h-g\left(\partial_{\tau}\left(H_{A^{t}} h\right)\right) d \sigma \\
= & \int_{\partial \Omega}(\nu \cdot A \nabla v) h-g\left(\nu \cdot A^{t} \nabla w\right) d \sigma \\
= & \int_{\Omega} \operatorname{div}(A(w \nabla v))-\operatorname{div}\left(A^{t}(v \nabla w)\right) d x d y \\
= & \int_{\Omega} \nabla w \cdot A \nabla v-\nabla v \cdot A^{t} \nabla w d x d y=0 .
\end{aligned}
$$

The integrating by parts above in unbounded domains can be justified by the decay properties of $v$ and $w$, passing to the limit through bounded domains.

Combining these two facts we may now prove that

$$
\|\nu \cdot A \nabla u\|_{L^{p}(\partial \Omega)} \leq C(p)\left\|\partial_{\tau} f_{0}\right\|_{L^{p}(\partial \Omega)},
$$

for $u$ which solve (1.1) with boundary data $f_{0}$. We can do this using duality:

$$
\begin{aligned}
\left|\int_{\partial \Omega}(\nu \cdot A \nabla u) h d \sigma\right| & =\left|\int_{\partial \Omega}\left(\partial_{\tau}\left(H_{A} f_{0}\right)\right) h d \sigma\right| \\
& =\left|-\int_{\partial \Omega}\left(\partial_{\tau} f_{0}\right)\left(H_{A^{t}} h\right) d \sigma\right| \\
& \leq C(p)\left\|\partial_{\tau} f_{0}\right\|_{L^{p}(\partial \Omega)}\|h\|_{L^{p^{\prime}}(\partial \Omega)},
\end{aligned}
$$

so (2.14) follows. It is a simple exercise to show that $|\nu \cdot \nabla u| \leq C\left(|\nu \cdot A \nabla u|+\left|\partial_{\tau} u\right|\right)$ from which it is easy to deduce (2.11). We also have the reverse of (2.14):

$$
\left\|\partial_{\tau} u\right\|_{L^{p}(\partial \Omega)} \leq C(p)\|\nu \cdot \widetilde{A} \nabla u\|_{L^{p}(\partial \Omega)}
$$

for $u$ which satisfy (1.2) with boundary data $g_{0}$ and $A$ replaced by $\widetilde{A}$. This follows from (2.14), since the conormal derivative becomes the tangential derivative of the conjugate and the tangential derivative becomes the conormal derivative of the conjugate. As before we then deduce (2.12).

The following lemma 19 regarding a certain change of variables will be crucial in obtaining the boundedness of the layer potentials.

Lemma 2.10. Suppose $\Omega=\left\{(x, t) \in \mathbf{R}^{2} \mid \phi(x)<t\right\}$ is the domain above the graph of a Lipschitz function $\phi$. Let $A=A(x)$ be any matrix satisfying the ellipticity condition (1.3) and with coefficients independent of the vertical direction. Also suppose that $\operatorname{div} A \nabla u=0$ in $\Omega$. Then there exists a change of variables $\Phi: \Omega^{\prime} \rightarrow \Omega$ such that

(i) If $v=u \circ \Phi$, then $\operatorname{div} B \nabla v=0$ in $\Omega^{\prime}$, where $B$ is upper triangular and independent of the t-variable of the form

$$
B=\left(\begin{array}{ll}
1 & c \\
0 & d
\end{array}\right)
$$

(ii) The domain $\Omega^{\prime}$ is the domain above the graph of a Lipschitz function. 
Proof. The transformation $\Phi$ defined by $\Phi(y, s)=(f(y), s+g(y))$ does the job with $f^{-1}$ chosen to be the primitive of $1 / a_{11}$ and $g$ chosen to be the primitive of $a_{21} \circ f$, where $A=\left(a_{i j}\right)_{i j}$. We refer the reader to Lemma 3.47 in [19] for a detailed proof.

Remark 2.11. The same result holds with $B$ replaced by the lower triangular matrix $B^{t}$. The proof only needs to be modified by choosing $g$ to instead be the primitive of $a_{12} \circ f$.

Escauriaza observed (see [19, p. 250]) that the operator $\operatorname{div} B^{t} \nabla$, where $B$ is as in (2.15), can be written in non-divergence form. Indeed, since the coefficients only depend on the $x$-direction,

$$
\begin{aligned}
\operatorname{div} B^{t} \nabla u & =\partial_{x x} u(x, t)+\partial_{t}\left(c(x) \partial_{x} u(x, t)\right)+\partial_{t}\left(d(x) \partial_{t} u(x, t)\right) \\
& =\partial_{x x} u(x, t)+c(x) \partial_{x t} u(x, t)+d(x) \partial_{t t} u(x, t) .
\end{aligned}
$$

We remark that then $\left(u_{t}, u_{x}\right)$ is a conjugate pair, since $u_{t}$ is a solution and we have

$$
\begin{aligned}
\partial_{t}\left(u_{x}\right) & =\partial_{x}\left(u_{t}\right), \\
-\partial_{x}\left(u_{x}\right) & =c \partial_{x}\left(u_{t}\right)+d \partial_{t}\left(u_{t}\right),
\end{aligned}
$$

so in this case $\widetilde{u_{t}}=u_{x}$. Solutions to non-divergence form equations in $\mathbf{R}^{2}$ also enjoy additional regularity. This is stated explicitly as the lemma below, which is an immediate consequence of Theorem 11.3 in [13, Remark (3) which follows it, and the discussion in Section 11.2. (This can also be seen directly when the coefficients are $t$-independent, since both $u_{t}$ and $u_{x}$ solve divergence form equations. Clearly $u_{t}$ satisfies the same equation as $u$, and $u_{x}$ solves an equation of the same type since $\widetilde{u_{t}}=u_{x}$.)

Lemma 2.12. Let $u \in H^{1,2}\left(B_{r}\right)$ satisfy $\operatorname{div} B^{t} \nabla u=0$ with coefficient matrix $B^{t}$, with $B$ as in (2.15). Then there exist constants $C>0$ and $\alpha \in(0,1]$, depending only on $\lambda$ and $\Lambda$, such that

$$
|\nabla u(X)-\nabla u(Y)| \leq \frac{C}{r^{\alpha}}\left\|\partial_{t} u\right\|_{L^{\infty}\left(B_{r}\right)}|X-Y|^{\alpha}
$$

for all $X, Y \in B_{2 r / 3}$.

\section{Reduction to the Boundedness of LAYER Potentials}

The aim of this section is to reduce the proof of Theorem 1.4 to proving the boundedness on $L^{p}(\partial \Omega)(1<p<\infty)$ of the double layer potential

$$
\mathcal{K}(f)(X)=\lim _{h \searrow 0} \int_{\partial \Omega} \nu(Y) \cdot A^{t}(Y) \nabla \Gamma_{(x, \phi(x)+h)}(Y) f(Y) d \sigma(Y)
$$

and the related potential

$$
\mathcal{L}(f)(X)=\lim _{h \searrow 0} \int_{\partial \Omega} \tau(Y) \cdot \nabla \Gamma_{(x, \phi(x)+h)}(Y) f(Y) d \sigma(Y),
$$

where $X=(x, \phi(x)) \in \partial \Omega$. This will be done in three steps under the assumption that the coefficient matrix $A$ is of the form (2.15). First, we will show that the nontangential maximal function of the gradient of a solution can be controlled in the $L^{p_{-}}$ norm by the $L^{p}$-norms of the layer potentials of $u_{t}$ and its conjugate $\widetilde{u_{t}}$. Secondly, we will show that $\left\|\widetilde{u_{t}}\right\|_{L^{p}(\partial \Omega)} \leq C(p)\|\nabla u\|_{L^{p}(\partial \Omega)}$. Finally, it is straightforward to combine these results with Theorem 2.9 and Lemma 2.10 to achieve our aim. 
Lemma 2.10 shows us that for the proof of Theorem 1.4, we may assume $A$ is of the form (2.15) without loss of generality, so let us fix, once and for all, $\Gamma_{X}$ to be the fundamental solution of the operator $L=\operatorname{div} A \nabla \cdot$ with pole at $X$, where $A$ is of this form. With this choice $\Gamma_{X}$ is a solution to a non-divergence form elliptic equation $L^{t} \Gamma_{X}=0$ away from $X$. Throughout the next two sections we will make the following a priori assumptions: $A=I$ for large $x, A$ and $\phi$ are smooth functions, $\phi^{\prime} \equiv \alpha_{0}$ for $x$ large, and $x \mapsto \phi(x)-\alpha_{0} x \in C_{0}^{\infty}$. Once the theorems here have been proved under our a priori assumptions it is a simple matter to obtain the general case.

Theorem 3.1. Let $\Omega=\left\{(x, t) \in \mathbf{R}^{2} \mid \phi(x)<t\right\}$ for some Lipschitz function $\phi$, $\left\|\phi^{\prime}\right\|_{L^{\infty}(\mathbf{R})} \leq k$. Let $L=\operatorname{div} A \nabla$ be an elliptic operator satisfying (1.3) with coefficient matrix $A=A(x)$ of measurable functions bounded by $\Lambda$ independent of the t-variable and of the form (2.15). Then for each $p>1$ there exists a constant $C(p)$, depending only on $\lambda, \Lambda, k$ and $p$, such that for any function $u: \Omega \rightarrow \mathbf{R}$ such that $L u=0$, and $u(X)=O\left(|X|^{\delta-1}\right)$ and $|\nabla u(X)|=O\left(|X|^{\delta-2}\right)$ for all $\delta>0$ as $|X| \rightarrow \infty$, we have

$$
\|\tilde{N}(\nabla u)\|_{L^{p}(\partial \Omega)} \leq C(p)\left(\|\nabla u\|_{L^{p}(\partial \Omega)}+\left\|\mathcal{K}\left(u_{t}\right)\right\|_{L^{p}(\partial \Omega)}+\left\|\mathcal{L}\left(\tilde{u}_{t}\right)\right\|_{L^{p}(\partial \Omega)}\right) .
$$

Proof. Firstly, exactly as in the proof of Lemma 8.10 in [21], we will estimate $\tilde{N}(\nabla u)$ pointwise by the maximal function of $N\left(u_{t}\right)$ and $\partial_{\tau} u$, so to prove the lemma we will then need to control $N\left(u_{t}\right)$ in the $L^{p}$-norm. This can be done by using an idea of Verchota and Vogel 26] to write $u_{t}$ as the sum of two potentials.

Fix $Q \in \partial \Omega$ and an $X \in \Gamma(Q)$. For $Z=(z, r) \in B_{\frac{3}{4} \delta(X)}(X)$, let $Z^{*}=(z, \phi(z))$ be the vertical projection onto the boundary of $\Omega$. Choose $m$ so that $\left(B_{\frac{3}{4} \delta(X)}(X)\right)^{*} \subset$ $\Delta_{m \delta(X)}(Q) \equiv \Delta$. For any $c_{B} \in \mathbf{R}$, Lemmata 2.1 and 2.2 give

$$
\left(f_{B(X, \delta(X) / 2)}|\nabla u|^{2}\right)^{\frac{1}{2}} \leq \frac{C}{\delta(X)} f_{B(X, 3 \delta(X) / 4)}\left|u-c_{B}\right| .
$$

Choosing $c_{B}=f_{\Delta} u$ we have

$$
\left|u(Z)-f_{\Delta} u\right| \leq\left|u(Z)-u\left(Z^{*}\right)\right|+\left|u\left(Z^{*}\right)-f_{\Delta} u\right| .
$$

Now,

$$
f_{B(X, 3 \delta(X) / 4)}\left|u\left(Z^{*}\right)-f_{\Delta} u\right| \leq C f_{\Delta}\left|u\left(Z^{*}\right)-f_{\Delta} u\right| d \sigma \leq C \delta(X) f_{\Delta}\left|\partial_{\tau} u\right|
$$

by Poincaré's inequality on $\partial \Omega$, where $\partial_{\tau}$ is the tangential derivative along $\partial \Omega$, and also $\left|u(Z)-u\left(Z^{*}\right)\right| \leq C \delta(X) N\left(u_{t}\right)\left(Z^{*}\right)$. Therefore, combining these estimates, we have

$$
\tilde{N}(\nabla u)(Q) \leq C\left(M\left(\partial_{\tau} u\right)(Q)+M\left(N\left(u_{t}\right)\right)(Q)\right),
$$

where $M$ is the Hardy-Littlewood maximal operator, and so, for each $p>1$,

$$
\|\widetilde{N}(\nabla u)\|_{L^{p}(\partial \Omega)} \leq C(p)\left(\|\nabla u\|_{L^{p}(\partial \Omega)}+\left\|N\left(u_{t}\right)\right\|_{L^{p}(\partial \Omega)}\right) .
$$

Now to estimate the last term above, we wish to find a representation for $u_{t}$. Recall Green's second identity: Let us write $L=\operatorname{div} A \nabla$ and $L^{t}=\operatorname{div} A^{t} \nabla$. Then we have

$$
\int_{\Omega}\left((L u) v-u\left(L^{t} v\right)\right)=\int_{\partial \Omega}\left((\nu \cdot A \nabla u) v-\left(\nu \cdot A^{t} \nabla v\right) u\right) d \sigma
$$


so, for $u$ such that $L u=0$ and replacing $v$ with the fundamental solution $\Gamma_{X}$ for $L$, so that $L^{t} \Gamma_{X}=\delta_{X}$, the Dirac mass at $X$, we obtain

$$
u(X)=\int_{\partial \Omega}\left(\left(\nu \cdot A^{t} \nabla \Gamma_{X}\right) u-(\nu \cdot A \nabla u) \Gamma_{X}\right) d \sigma .
$$

Since the elements of $A$ only depend on the $x$-variable $u_{t}$ is also a solution and so we have

$$
u_{t}(X)=\int_{\partial \Omega}\left(\left(\nu \cdot A^{t} \nabla \Gamma_{X}\right) u_{t}-\left(\nu \cdot A \nabla u_{t}\right) \Gamma_{X}\right) d \sigma .
$$

Recall that $\nu \cdot A \nabla u_{t}=\tau \cdot \nabla \widetilde{u_{t}}$, where $\widetilde{u_{t}}$ is the conjugate of $u_{t}$. Therefore,

$$
\begin{aligned}
u_{t}(X) & =\int_{\partial \Omega}\left(\left(\nu \cdot A^{t} \nabla \Gamma_{X}\right) u_{t}-\left(\partial_{\tau}\left(\widetilde{u}_{t}\right)\right) \Gamma_{X}\right) d \sigma \\
& =\int_{\partial \Omega}\left(\left(\nu \cdot A^{t} \nabla \Gamma_{X}\right) u_{t}+\tau \cdot \nabla \Gamma_{X}\left(\widetilde{u}_{t}\right)\right) d \sigma .
\end{aligned}
$$

To complete the proof we will estimate $N\left(u_{t}\right)$ by estimating each term on the right-hand side of (3.2). This can be done using the singular integral representation we obtain for $\mathcal{K}$ and $\mathcal{L}$ in Section 4 , and so we leave the end of the proof to be completed in Remark 4.4. It is in this step where we will use the hypothesis that $A$ is of the form (2.15).

Lemma 3.2. Let $u, \Omega$ and $A$ be as in Theorem 3.1. There exists a constant $C(p)>0$ depending only on $\lambda, \Lambda, k$ and $p$ such that

$$
\left\|\widetilde{u_{t}}\right\|_{L^{p}(\partial \Omega)} \leq C(p)\|\nabla u\|_{L^{p}(\partial \Omega)} .
$$

Proof. This would be immediate if it were true that $A=B^{t}$, where $B$ is as in (2.15), since, for a solution $v$ to $\operatorname{div} B^{t} \nabla v=0,\left(v_{t}, v_{x}\right)$ is a conjugate pair (see Section 2). For our case we can use the change of variables $\Phi$ from Lemma 2.10 (and Remark 2.11), so $v=u \circ \Phi$ and $B^{t}=\left(\operatorname{det} \Phi^{\prime}\right)\left(\Phi^{\prime-1}\right)^{t} A \Phi^{\prime-1}$. A simple calculation reveals that taking derivatives in the $t$-variable commutes with the transformation $\Phi^{-1}$. Also the explicit form of the transformation shows us that $t$-derivatives of $\widetilde{u}$ are comparable to $t$-derivatives of $\widetilde{v}$ and $\|\nabla u\|_{L^{p}(\partial \Omega)} \simeq\|\nabla v\|_{L^{p}\left(\partial \Omega^{\prime}\right)}$. Finally we claim conjugation also commutes with the transformation $\Phi^{-1}$. Proving this means showing

$$
\widetilde{v} \circ \Phi^{-1}=\left(v \circ \Phi^{-1}\right)^{\sim} .
$$

With $v_{0}:=\widetilde{u} \circ \Phi$, we have that

$$
\nabla v=\Phi^{\prime}(\nabla u \circ \Phi) \text { and } \nabla v_{0}=\Phi^{\prime}(\nabla \widetilde{u} \circ \Phi),
$$

so using (2.9), we obtain

$$
\left(\begin{array}{cc}
0 & 1 \\
-1 & 0
\end{array}\right)\left(\Phi^{\prime}\right)^{-1} \nabla v_{0}=A\left(\Phi^{\prime}\right)^{-1} \nabla v .
$$

Multiplying on the left by $\left(\Phi^{\prime-1}\right)^{t}$ we see that

$$
\left(\operatorname{det} \Phi^{\prime}\right)^{-1}\left(\begin{array}{cc}
0 & 1 \\
-1 & 0
\end{array}\right) \nabla v_{0}=\left(\Phi^{\prime-1}\right)^{t} A\left(\Phi^{\prime}\right)^{-1} \nabla v .
$$

Thus $\left(v, v_{0}\right)$ solves (2.9) with $A$ replaced with $B^{t}$, that is, $v_{0}=\widetilde{v}$. The last equality can be rewritten $\widetilde{u} \circ \Phi=(u \circ \Phi)^{\sim}$ from which (3.3) follows. 
These observations allow us to conclude that

$$
\begin{aligned}
& \left\|\widetilde{u_{t}}\right\|_{L^{p}(\partial \Omega)}=\left\|\left(\partial_{t}\left(v \circ \Phi^{-1}\right)\right)^{\sim}\right\|_{L^{p}(\partial \Omega)}=\left\|\partial_{t}\left(\widetilde{v} \circ \Phi^{-1}\right)\right\|_{L^{p}(\partial \Omega)} \\
& \leq C(p)\left\|\partial_{t} \widetilde{v}\right\|_{L^{p}\left(\partial \Omega^{\prime}\right)}=C(p)\left\|\widetilde{v}_{t}\right\|_{L^{p}\left(\partial \Omega^{\prime}\right)} \leq C(p)\|\nabla v\|_{L^{p}\left(\partial \Omega^{\prime}\right)} \simeq\|\nabla u\|_{L^{p}(\partial \Omega)},
\end{aligned}
$$

and so the lemma is proved.

Once we know $\mathcal{K}$ and $\mathcal{L}$ are bounded for all $A$ of the form (2.15), we have from Theorems 3.1 and 3.2 that $\|\tilde{N}(\nabla u)\|_{L^{p}(\partial \Omega)} \leq C(p)\|\nabla u\|_{L^{p}(\partial \Omega)}(1<p<\infty)$. Using Lemma 2.10 we extend this to arbitrary $A$. One then applies Theorems 2.8 and 2.9 to conclude the proof of Theorem 1.4. It remains to demonstrate the boundedness of $\mathcal{K}$ and $\mathcal{L}$ on $L^{p}$. This task will occupy us for the next two sections.

\section{BOUNDEDNESS OF THE LAYER POTENTIALS ON BOUNDARIES WITH SMALL LIPSCHITZ CONSTANTS}

In this section we will show that $\mathcal{K}$ and $\mathcal{L}$ are bounded on $L^{p}(\partial \Omega)(1<p<\infty)$ in the special case that $\Gamma_{X}$ is the fundamental solution of the operator $L=\operatorname{div}(A \nabla \cdot)$ with pole at $X$, where $A$ is of the form (2.15) and that our domain $\Omega$ has a boundary which is close to linear in the sense that, for $\alpha_{0} \in[-k, k],\left\|\phi^{\prime}-\alpha_{0}\right\|_{L^{\infty}(\mathbf{R})} \leq \varepsilon_{0}$, where $\varepsilon_{0}>0$ depends only on the ellipticity constants and $k>0$. Lemma 2.10 shows that the first assumption can be made without loss of generality. However, if we are to succeed in proving Theorem 1.4, the second assumption must be dropped. This will be done in Section 5. Denote by $\Lambda^{k}\left(\varepsilon_{0}\right)$ the set of all Lipschitz functions $\phi$ such that $\left\|\phi^{\prime}-\alpha_{0}\right\|_{L^{\infty}(\mathbf{R})} \leq \varepsilon_{0}$, with $\alpha_{0} \in[-k, k]$. We will always require that $0<\varepsilon_{0} \leq k$, so the Lipschitz constant of $\phi \in \Lambda^{k}\left(\varepsilon_{0}\right)$ is no more than $2 k$. We remind the reader of the a priori smoothness assumptions made at the beginning of Section 3 ,

Lemma 4.1. There exists constants $C_{3}>0$ and $0<\alpha \leq 1$, depending only on $\lambda$ and $\Lambda$, such that

$$
\left|\nabla \Gamma_{X}(Y)\right| \leq \frac{C_{3}}{|X-Y|}
$$

for all $X, Y \in \mathbf{R}^{2}, X \neq Y$,

$$
\left|\nabla \Gamma_{X}(Y)-\nabla \Gamma_{X^{\prime}}(Y)\right| \leq \frac{C_{3}\left|X-X^{\prime}\right|^{\alpha}}{\left(|X-Y|+\left|X^{\prime}-Y\right|\right)^{1+\alpha}}
$$

when $\left|X-X^{\prime}\right| \leq \frac{1}{2} \max \left\{|X-Y|,\left|X^{\prime}-Y\right|\right\}$, and

$$
\left|\nabla \Gamma_{X}(Y)-\nabla \Gamma_{X}\left(Y^{\prime}\right)\right| \leq \frac{C_{3}\left|Y-Y^{\prime}\right|^{\alpha}}{\left(|X-Y|+\left|X-Y^{\prime}\right|\right)^{1+\alpha}}
$$

when $\left|Y-Y^{\prime}\right| \leq \frac{1}{2} \max \left\{|X-Y|,\left|X-Y^{\prime}\right|\right\}$.

Proof. Fix $X, Y \in \mathbf{R}^{2}, X \neq Y$ and set $r=|X-Y|$. Caccioppoli's inequality (Lemma 2.1) gives us that

$$
f_{B_{r / 4}(Y)}\left|\nabla \Gamma_{X}(Z)\right|^{2} d Z \leq \frac{C}{r^{2}} f_{B_{r / 2}(Y)}\left|\Gamma_{X}(Z)\right|^{2} d Z .
$$


Now we apply a linear change of variables $\Phi$ which sends $0=(0,0)$ to $X$ and $\left(0,-R_{1} / 4\right)$ to $Y$ :

$$
\begin{aligned}
& \frac{C}{r^{2}} f_{B_{\frac{r}{2}(Y)}}\left|\Gamma_{X}(Z)\right|^{2} d Z=\frac{C}{r^{2}} f_{B_{\frac{1}{8} R_{1}}\left(\left(0,-R_{1} / 4\right)\right)}\left|\Gamma_{\Phi(0)} \circ \Phi(Z)\right|^{2} d Z \\
& \leq \frac{C}{r^{2}} f_{B_{\frac{1}{8} R_{1}}\left(\left(0,-R_{1} / 4\right)\right)}|\log (|Z|)|^{2} d Z \leq \frac{C}{r^{2}},
\end{aligned}
$$

where the second to last inequality follows from Theorem [2.6. since $\Gamma_{\Phi(0)} \circ \Phi$ is the fundamental solution to an elliptic operator with the same constants $\lambda$ and $\Lambda$ and pole at the origin. Now $\partial_{s} \Gamma_{X}(y, s)$ satisfies $L\left(\partial_{s} \Gamma_{X}\right)=0$ away from $X$ since the coefficients do not depend on the second variable; thus, using the above and Lemma 2.2 we have

$$
\sup _{B_{r / 8}(Y)}\left|\partial_{s} \Gamma_{X}\right| \leq\left(f_{B_{r / 4}(Y)}\left|\nabla \Gamma_{X}(Z)\right|^{2} d Z\right)^{\frac{1}{2}} \leq \frac{C}{r} .
$$

This last bound shows we may now apply Lemma 2.12 with $B_{r}$ replaced with $B_{r / 8}(Y)$ to obtain

$$
\begin{aligned}
\left|\partial_{y} \Gamma_{X}(Y)\right| & \leq\left|\partial_{y} \Gamma_{X}(Z)\right|+\left|\partial_{y} \Gamma_{X}(Y)-\partial_{y} \Gamma_{X}(Z)\right| \\
& \leq\left|\partial_{y} \Gamma_{X}(Z)\right|+\frac{C}{r}\left(\frac{|Y-Z|}{r}\right)^{\alpha} \\
& \leq\left|\partial_{y} \Gamma_{X}(Z)\right|+C / r
\end{aligned}
$$

for $Z \in B_{r / 8}(Y)$. Averaging this inequality over $Z \in B_{r / 8}(Y)$, and using (4.4) and (4.5) with $r$ replaced with $r / 2$ yields

$$
\left|\partial_{y} \Gamma_{X}(Y)\right| \leq \frac{C}{r} .
$$

Combining this with (4.6) yields (4.1). The same application of Lemma 2.12 now easily yields (4.3): with $r=\max \left(|Y-X|,\left|Y^{\prime}-X\right|\right)=|Y-X|$, say, we have for $\left|Y-Y^{\prime}\right| \leq r / 2$ that

$$
\begin{aligned}
\left|\nabla \Gamma_{X}(Y)-\nabla \Gamma_{X}\left(Y^{\prime}\right)\right| & \leq \frac{C}{r^{\alpha}}\left\|\nabla \Gamma_{X}\right\|_{L^{\infty}\left(B_{3 r / 4}(Y)\right)}\left|Y-Y^{\prime}\right|^{\alpha} \\
& \leq \frac{C}{r^{1+\alpha}}\left|Y-Y^{\prime}\right|^{\alpha} .
\end{aligned}
$$

Inequality (4.2) follows from the fact (Lemma 2.7) that $X \mapsto \Gamma_{X}(Y)$ is a solution to an elliptic equation in divergence form, so we may use the standard Hölder continuity result of Lemma 2.4 now with $r=\max \left(|X-Y|,\left|X^{\prime}-Y\right|\right)=|X-Y|$, say, we have for $\left|X-X^{\prime}\right| \leq r / 2$ that

$$
\begin{aligned}
\left|\nabla \Gamma_{X}(Y)-\nabla \Gamma_{X^{\prime}}(Y)\right| & \leq \frac{C}{r^{\alpha}}\left\|\nabla \Gamma_{X}\right\|_{L^{2}\left(B_{3 r / 4}(Y)\right)}\left|X-X^{\prime}\right|^{\alpha} \\
& \leq \frac{C}{r^{1+\alpha}}\left|X-X^{\prime}\right|^{\alpha},
\end{aligned}
$$

which completes the proof of the lemma. 
Let $\phi \in \Lambda^{\frac{k}{4}}\left(\varepsilon_{0}\right)$ be a Lipschitz function with $\left\|\phi^{\prime}-\alpha_{0}\right\|_{L^{\infty}(\mathbf{R})}=\varepsilon \leq \varepsilon_{0}, \Omega=$ $\Omega^{+}=\{(x, t) \mid t>\phi(x)\}$ and $\Omega^{-}=\{(x, t) \mid t<\phi(x)\}$, and write $\partial \Omega_{h}=\partial \Omega+(0, h)$ and

$$
\left(\begin{array}{c}
k_{1, h}(x, y) \\
k_{2, h}(x, y)
\end{array}\right)=\nabla \Gamma_{(x, \phi(x)+h)}(y, \phi(y))
$$

for $h \in \mathbf{R}$. Define the function $K_{h}: \mathbf{R}^{2} \rightarrow \mathcal{M}$ by

$$
K_{h}=\left(\begin{array}{cc}
k_{1, h} & k_{2, h} \\
k_{1, h} & k_{2, h}
\end{array}\right) .
$$

We will write $K_{0}=K$. We have the following easy corollary to Lemma 4.1

Corollary 4.2. For each $\phi \in \Lambda^{\frac{k}{4}}\left(\varepsilon_{0}\right)$, the function $K: \mathbf{R}^{2} \rightarrow \mathcal{M}$ is a CalderónZygmund kernel. That is to say, there exist constants $C_{4}=C_{4}(k)>0$ and $\alpha \in$ $(0,1]$, depending only on $\lambda, \Lambda$ and the Lipschitz constant $k$, such that (2.1), (2.2) and (2.3) hold with $C=C_{4}$.

For Banach spaces $\mathcal{X}$ and $\mathcal{Y}$ of real matrix-valued functions on $\mathbf{R}$, define $T: \mathcal{X} \rightarrow$ $\mathcal{Y}^{\prime}$ by

$$
\langle G, T(F)\rangle=\lim _{h \searrow 0} \int_{\mathbf{R}^{2}} G(x)^{t} K_{h}(x, y) F(y) d y d x,
$$

where $F \in \mathcal{X}$ and $G \in \mathcal{Y}$ and $\langle\cdot, \cdot\rangle$ is the dual pairing in $\mathcal{Y}$. The transpose operator $T^{t}$ is defined as $\left\langle F, T^{t}(G)\right\rangle=\langle G, T(F)\rangle^{t}$. Our aim will be to show that $T$ extends to a bounded operator on $L^{2}\left(\mathbf{R}^{2}, \mathcal{M}\right)$, but first we must choose appropriate Banach spaces, $\mathcal{X}$ and $\mathcal{Y}$, and show this definition makes sense. This is the content of the following lemma, and for that we will need the following notation. We denote by $\mathcal{S}=\mathcal{S}(\mathbf{R}, \mathcal{M})$ the space of Schwarz functions on $\mathbf{R}$ with values in $\mathcal{M}$ and for a function $B_{0}: \mathbf{R} \rightarrow \mathcal{M}, B_{0} \mathcal{S}$ is the set of all functions obtained by multiplying functions in $\mathcal{S}$ on the left by $B_{0}$. Let $\delta$ be the distance function $\delta(X)=\operatorname{dist}(X, \partial \Omega)$. The spaces $C_{0}^{\infty}(\mathbf{R}, \mathbf{R})$ and $C_{0}^{\infty}=C_{0}^{\infty}(\mathbf{R}, \mathcal{M})$ are the spaces of compactly supported smooth functions from $\mathbf{R}$ to $\mathbf{R}$ or $\mathcal{M}$ respectively. Set $B_{1}: \mathbf{R} \rightarrow \mathcal{M}$ equal to the matrix with first column being $\left(1+\left(\phi^{\prime}\right)^{2}\right)^{\frac{1}{2}} A \nu$ and second column $\left(1+\left(\phi^{\prime}\right)^{2}\right)^{\frac{1}{2}} \tau$. Set $B_{2}: \mathbf{R} \rightarrow \mathcal{M}$ equal to the diagonal matrix with both diagonal entries being $\left(1+\left(\phi^{\prime}\right)^{2}\right)^{\frac{1}{2}} \nu \cdot A^{t} \kappa^{\perp}$, where $\kappa^{\perp}=\left(-\alpha_{0}, 1\right)$, and set $B_{3}: \mathbf{R} \rightarrow \mathcal{M}$ equal to the diagonal matrix with both diagonal entries being $\left(1+\left(\phi^{\prime}\right)^{2}\right)^{\frac{1}{2}} \tau \cdot \kappa$, where $\kappa=\left(1, \alpha_{0}\right)$.

Note that by the ellipticity condition (1.3), for sufficiently small $\varepsilon_{0}$ (depending only on the ellipticity constants and $k), B_{1}, B_{2}$ and $B_{3}$ are invertible with inverses bounded in the $L^{\infty}(\mathbf{R}, \mathcal{M})$-norm in terms of the ellipticity constants and $k$.

Lemma 4.3. For each $\phi \in \Lambda^{\frac{k}{4}}\left(\varepsilon_{0}\right)$, the operator $T$ is a continuous linear operator from $B_{1} \mathcal{S}$ to $\left(B_{0} \mathcal{S}\right)^{\prime}$, for any bounded $B_{0}$.

Proof. We fix $f, g \in \mathcal{S}(\mathbf{R}, \mathbf{R}), h \in C_{0}^{\infty}(\mathbf{R}, \mathbf{R})$ with $h$ positive and equal to one near zero and define $u, v: \Omega \rightarrow \mathbf{R}$ by

$$
u(X)=f(x) h(t-\phi(x)) \text { and } v(X)=g(x) h(t-\phi(x)) .
$$

Green's first identity gives us that

$$
u(X)=-\int_{\Omega} \nabla u(Y) \cdot A^{t}(Y) \nabla \Gamma_{X}(Y) d Y+\int_{\partial \Omega} u(Y) \nu(Y) \cdot A^{t}(Y) \nabla \Gamma_{X}(Y) d \sigma(Y) .
$$


By multiplying by a bounded function $b$ and the function $v$, and integrating, we obtain

$$
\begin{aligned}
& \int_{\partial \Omega_{h}} u(X) b(X) v(X) d X \\
& =\int_{\partial \Omega_{h}}\left(-\int_{\Omega} \nabla u(Y) \cdot A^{t}(Y) \nabla \Gamma_{X}(Y) d Y\right) b(X) v(X) d \sigma(X) \\
& \quad+\int_{\partial \Omega_{h}} \int_{\partial \Omega} u(Y) \nu(Y) \cdot A^{t}(Y) \nabla \Gamma_{X}(Y) b(X) v(X) d \sigma(Y) d \sigma(X) .
\end{aligned}
$$

The left-hand side can easily be controlled by the bound on $b$ and the product of semi-norms $[f]_{1}^{0}[g]_{1}^{0}$, where

$$
[f]_{N}^{\beta}=\sup _{X}(1+|X|)^{N}\left|\partial^{\beta} f(X)\right|
$$

for $N \in \mathbf{Z}$ and $\beta \in \mathbf{N}$. We can control the first term on the right-hand side similarly. Indeed, observe that

$$
\begin{aligned}
\left|\int_{\Omega} \nabla u(Y) \cdot A^{t}(Y) \nabla \Gamma_{X}(Y) d Y\right| & \leq C\left([f]_{3}^{0}+[f]_{3}^{1}\right) \int_{\mathbf{R}^{2}} \frac{(1+|Y|)^{-3}}{|X-Y|} d Y \\
& \leq C\left([f]_{3}^{0}+[f]_{3}^{1}\right)
\end{aligned}
$$

and then proceed as before. So, we conclude the remaining term on the right-hand side is also controlled by a finite sum of products $\|b\|_{L^{\infty}(\mathbf{R})}[f]_{N}^{\beta}[g]_{M}^{\gamma}$, that is,

$$
\begin{aligned}
& \mid \int_{\partial \Omega_{h}} \int_{\partial \Omega} u(Y) \nu(Y) \cdot A^{t}(Y) \nabla \Gamma_{X}(Y) b(X) v(X) d \sigma(Y) d \sigma(X) \mid \\
& \leq C\|b\|_{L^{\infty}(\mathbf{R})} \sum_{N, M, \beta, \gamma}[f]_{N}^{\beta}[g]_{M}^{\gamma} .
\end{aligned}
$$

We may also compute, setting $R_{3}=R_{1} /\left(1+k^{2}\right)^{\frac{1}{2}}$ (where $R_{1}$ is the constant appearing in Theorem 2.6),

$$
\begin{aligned}
& \left|\int_{\partial \Omega}\left(\tau(Y) \cdot \nabla \Gamma_{X}(Y)\right) u(Y) d \sigma(Y)\right| \\
= & \left|\int_{\mathbf{R}}\left\{\partial_{1} \Gamma_{(x, \phi(x))}(y, \phi(y))+\phi^{\prime}(y) \partial_{2} \Gamma_{(x, \phi(x))}(y, \phi(y))\right\} u(Y) d y\right| \\
= & \left|\int_{\mathbf{R}} \frac{d}{d y}\left\{\Gamma_{(x, \phi(x))}(y, \phi(y))\right\} u(y, \phi(y)) d y\right| \\
\leq & \left|\int_{\mathbf{R} \backslash\left(x-R_{3}, x+R_{3}\right)} \frac{d}{d y}\left\{\Gamma_{(x, \phi(x))}(y, \phi(y))\right\} u(y, \phi(y)) d y\right| \\
& +\left|\int_{\left(x-R_{3}, x+R_{3}\right)} \Gamma_{(x, \phi(x))}(y, \phi(y)) \frac{d}{d y} u(y, \phi(y)) d y\right| \\
& +\sum_{Y \in\left\{x-R_{3}, x+R_{3}\right\}}\left|\Gamma_{(x, \phi(x))}(y, \phi(y)) u(y, \phi(y))\right| \\
\leq & C\left([f]_{2}^{0}+[f]_{0}^{1}+[f]_{0}^{0}\right),
\end{aligned}
$$


so allowing us to conclude

$$
\begin{array}{r}
\left|\int_{\partial \Omega_{h}} \int_{\partial \Omega} u(Y) \tau(Y) \cdot \nabla \Gamma_{X}(Y) b(X) v(X) d \sigma(Y) d \sigma(X)\right| \\
\leq C\|b\|_{\infty} \sum_{N, M, \beta, \gamma}[f]_{N}^{\beta}[g]_{M}^{\gamma} .
\end{array}
$$

However,

$$
K_{h}(x, \cdot) B_{1}=\left(\begin{array}{cc}
\left(1+\phi^{\prime}\right)^{\frac{1}{2}} \nu \cdot A^{t} \nabla \Gamma_{(x, \phi(x)+h)} & \left(1+\phi^{\prime}\right)^{\frac{1}{2}} \tau \cdot \nabla \Gamma_{(x, \phi(x)+h)} \\
\left(1+\phi^{\prime}\right)^{\frac{1}{2}} \nu \cdot A^{t} \nabla \Gamma_{(x, \phi(x)+h)} & \left(1+\phi^{\prime}\right)^{\frac{1}{2}} \tau \cdot \nabla \Gamma_{(x, \phi(x)+h)}
\end{array}\right) .
$$

Therefore, by writing in (4.8),

$$
G(x)^{t} K_{h}(x, y) F(y)=G_{\infty}(x)^{t} B_{0}(x)^{t} K_{h}(x, y) B_{1}(y) F_{\infty}(y),
$$

with $F_{\infty} \in \mathcal{S}$ and $G_{\infty} \in \mathcal{S}$, we see that each matrix element in (4.8) is a sum of terms of the form of either (4.11) or (4.12). Thus provided the limit exists, (4.8) is controlled by a finite sum of $\left[F_{\infty}\right]_{N}^{\beta}\left[G_{\infty}\right]_{M}^{\gamma}$, with the semi-norms defined analogously to (4.10). We can easily show the limit in (4.8) exists almost everywhere under our a priori assumptions: By the above it suffices to show both

$$
\int_{\partial \Omega} \nu(Y) \cdot A^{t}(Y) \nabla \Gamma_{X}(Y) u(Y) d \sigma(Y)
$$

and

$$
\int_{\partial \Omega} \tau(Y) \cdot \nabla \Gamma_{X}(Y) u(Y) d \sigma(Y)
$$

converge uniformly in $x$ as $h \searrow 0$, where $X=(x, \phi(x)+h)$. To show (4.13) converges we write

$$
\begin{aligned}
& \int_{\partial \Omega} \nu(Y) \cdot A^{t}(Y) \nabla \Gamma_{X}(Y) u(Y) d \sigma(Y) \\
& =\int_{\partial \Omega \cap B_{1}(X)} \nu(Y) \cdot A^{t}(Y) \nabla \Gamma_{X}(Y) u(Y) d \sigma(Y) \\
& \quad+\int_{\partial \Omega \backslash B_{1}(X)} \nu(Y) \cdot A^{t}(Y) \nabla \Gamma_{X}(Y) u(Y) d \sigma(Y) .
\end{aligned}
$$

The second integral on the right-hand side clearly converges uniformly in $x$. To show that the first also does, we can use the divergence theorem to see it is equal to

$$
\begin{aligned}
& \int_{\partial \Omega \cap B_{1}(X)} \nu(Y) \cdot A^{t}(Y) \nabla \Gamma_{X}(Y)(u(Y)-u(X)) d \sigma(Y) \\
& \quad+u(X) \int_{\partial \Omega \cap B_{1}(X)} \nu(Y) \cdot A^{t}(Y) \nabla \Gamma_{X}(Y) d \sigma(Y) \\
& =\int_{\partial \Omega \cap B_{1}(X)} \nu(Y) \cdot A^{t}(Y) \nabla \Gamma_{X}(Y)(u(Y)-u(X)) d \sigma(Y) \\
& \quad-u(X) \int_{\Omega^{-} \cap \partial B_{1}(X)} \nu(Y) \cdot A^{t}(Y) \nabla \Gamma_{X}(Y) d \sigma(Y),
\end{aligned}
$$


and both integrals on the right-hand side converge uniformly in $x$ under our a priori smoothness assumptions. We can see (4.14) converges similarly: again we write

$$
\begin{aligned}
\int_{\partial \Omega} \tau(Y) \cdot \nabla \Gamma_{X}(Y) u(Y) d \sigma(Y)= & \int_{\partial \Omega \cap B_{1}(X)} \tau(Y) \cdot \nabla \Gamma_{X}(Y) u(Y) d \sigma(Y) \\
& +\int_{\partial \Omega \backslash B_{1}(X)} \tau(Y) \cdot \nabla \Gamma_{X}(Y) u(Y) d \sigma(Y) .
\end{aligned}
$$

As before, the second integral on the right-hand side converges uniformly in $x$. The Fundamental Theorem of Calculus shows that the first is equal to

$$
\begin{aligned}
& \int_{\partial \Omega \cap B_{1}(X)} \tau(Y) \cdot \nabla \Gamma_{X}(Y)(u(Y)-u(X)) d \sigma(Y) \\
& \quad+u(X) \int_{\partial \Omega \cap B_{1}(X)} \tau(Y) \cdot \nabla \Gamma_{X}(Y) d \sigma(Y) \\
& =\int_{\partial \Omega \cap B_{1}(X)} \tau(Y) \cdot \nabla \Gamma_{X}(Y)(u(Y)-u(X)) d \sigma(Y) \\
& \quad-u(X) \int_{\Omega^{-} \cap \partial B_{1}(X)} \tau(Y) \cdot \nabla \Gamma_{X}(Y) d \sigma(Y),
\end{aligned}
$$

and once again both integrals on the right-hand side converge uniformly in $x$.

Since we clearly have the representation (2.4), what we have shown so far is that $T$ is a singular integral operator associated to the Calderón-Zygmund kernel $K$.

Remark 4.4. This singular integral representation can be used to complete the proof of Lemma 3.1 since $\mathcal{K}$ and $\mathcal{L}$ can be easily written in terms of $T$ and so using (3.2) can control $N\left(u_{t}\right)$. We define

$$
\mathcal{T}(F)(Z)=\int_{\mathbf{R}} K_{r}(z, y) F(y) d y,
$$

for $(z, r)=Z \in \Omega$, and

$$
T^{(\delta)}(F)(X)=\int_{|y-x| \geq \delta} K(x, y) F(y) d y,
$$

for $X \in \partial \Omega$. Fixing $(z, r)=Z \in \Gamma(X)$ we claim that $\left|T^{(\delta)}(F)(X)-\mathcal{T}(Z)\right| \leq$ $C M(F)(X)$ for $\delta=\delta(Z)$. We have

$$
\begin{aligned}
& \left|T^{(\delta)}(F)(X)-\mathcal{T}(Z)\right| \\
& =\left|\int_{|x-y| \geq \delta(Z)} K(x, y) F(y) d y-\int_{\mathbf{R}} K_{r}(z, y) F(y) d y\right| \\
& =\left|\int_{|x-y| \geq \delta(Z)}\left(K(x, y)-K_{r}(z, y)\right) F(y) d y-\int_{|x-y|<\delta(Z)} K_{r}(z, y) F(y) d y\right| \\
& \leq\left|\int_{|x-y| \geq \delta(Z)}\left(K(x, y)-K_{r}(z, y)\right) F(y) d y\right|+\left|\int_{|x-y|<\delta(Z)} K_{r}(z, y) F(y) d y\right| .
\end{aligned}
$$


The first term can be estimated using (4.2):

$$
\begin{aligned}
& \left|\int_{|x-y| \geq \delta(Z)}\left(K(x, y)-K_{r}(z, y)\right) F(y) d y\right| \leq C \int_{|x-y| \geq \delta(Z)} \frac{|x-z|^{\alpha}|F(y)|}{|x-y|^{1+\alpha}} d y \\
& \leq C(1+a)^{\alpha} \delta(Z)^{\alpha} \int_{|x-y| \geq \delta(Z)} \frac{|F(y)|}{|x-y|^{1+\alpha}} d y \leq C M(F)(x) .
\end{aligned}
$$

The second term is also easily controlled using (4.1):

$$
\left|\int_{|x-y|<\delta(Z)} K_{r}(z, y) F(y) d y\right| \leq \frac{C}{\delta(Z)} \int_{|x-y|<\delta(Z)}|F(y)| d y \leq C M(F)(x),
$$

which proves the claim. The claim tells us that $T^{(\delta(Z))}(F)(X)$ and $M(F)(X)$ control $\mathcal{T}(F)(Z)$, so $N(\mathcal{T})$ can be controlled in the operator norm by the operator norm of $T^{*}+M$. Standard Calderón-Zygmund theory (see, for example, [14) tells us that we can control $T^{*}$ in terms of $T$. Writing $\mathcal{K}$ and $\mathcal{L}$ in terms of $T$ and using (3.2) then completes the proof of Lemma 3.1.

Our aim now is to prove the following.

Theorem 4.5. For each $k>0$, there exists an $\varepsilon_{0}>0$ depending only on $k, \lambda$ and $\Lambda$, such that, for any $\phi \in \Lambda^{\frac{k}{4}}\left(\varepsilon_{0}\right)$, the singular integral operator $T$ admits a continuous extension to $L^{2}(\mathbf{R}, \mathcal{M})$ and therefore also to $L^{p}(\mathbf{R}, \mathcal{M})$ for all $1<p<\infty$ with norm depending only on $p, \lambda, \Lambda$ and $k$. Consequently the layer potentials $\mathcal{K}$ and $\mathcal{L}$, defined, for $X=(x, \phi(x)) \in \partial \Omega$, by

$$
\mathcal{K}(f)(X)=\lim _{h \searrow 0} \int_{\partial \Omega} \nu \cdot A^{t}(Y) \nabla \Gamma_{(x, \phi(x)+h)}(Y) f(Y) d \sigma(Y)
$$

and

$$
\mathcal{L}(f)(X)=\lim _{h \searrow 0} \int_{\partial \Omega} \tau \cdot \nabla \Gamma_{(x, \phi(x)+h)}(Y) f(Y) d \sigma(Y),
$$

are bounded linear operators on $L^{p}(\partial \Omega, \mathbf{R})(1<p<\infty)$ when $\Omega=\{X=(x, t) \mid t>$ $\phi(x)\}$.

This will be proved by applying a version of the $T(B)$-Theorem. Let us first state some standard definitions and fix some more notation.

Let $M_{B_{0}}$ denote the operator which multiplies on the left by a function $B_{0}: \mathbf{R} \rightarrow$ $\mathcal{M}$. By a normalised bump we mean a function $F=\left(f_{i j}\right)_{i j} \in \mathcal{S}$ with support in a ball with radius 10 such that

$$
[F]_{0}^{\beta}:=\sup _{i, j, x}\left|\partial^{\beta} f_{i j}(x)\right| \leq 1
$$

for $\beta=0,1,2$. For such an $F$ define $F_{R}=R^{-1} F(\cdot / R)$. An operator $T$ is said to satisfy the weak boundedness property if there exists a constant $C>0$ such that

$$
\left|\left\langle G_{R}, T F_{R}\right\rangle\right| \leq C R^{-1} \text {. }
$$

A function $f: \mathbf{R} \rightarrow \mathbf{R}$ is said to be of bounded mean oscillation, written $f \in$ $\mathrm{BMO}$, if we have

$$
\|f\|_{\mathrm{BMO}}:=\sup _{I} f_{I}\left|f(x)-f_{I} f\right| d x<\infty,
$$

where the supremum is taken over all intervals $I$. A function $a: \mathbf{R} \rightarrow \mathbf{R}$ is said to be an $H^{1}$ atom if there exists an interval $I \subset \mathbf{R}$ such that (i) $a$ is supported in 
$I$, (ii) $\|a\|_{L^{2}(\mathbf{R})} \leq|I|^{-\frac{1}{2}}$, and (iii) $\int a=0$. For a bounded function $B_{0} \in B_{1} C_{0}^{\infty}$, $T\left(B_{0}\right)$ may be interpreted as a matrix-valued function, whose matrix elements are functions in BMO in the following manner. We shall call a matrix-valued function whose matrix elements are $H^{1}$ atoms, or BMO functions, again an $H^{1}$ atom, or a BMO function, respectively. For each $H^{1}$ atom $A_{0} \in B_{2} C_{0}^{\infty}$ with support in a ball $B$, choose a smooth function $\eta: \mathbf{R} \rightarrow \mathbf{R}$ with compact support which is identically one on the double of the ball $2 B$. Then we define

$$
\left\langle A_{0}, T\left(B_{0}\right)\right\rangle:=\left\langle A_{0}, T\left(\eta B_{0}\right)\right\rangle+\int_{\mathbf{R}}\left[\int_{\mathbf{R}} A_{0}(x) K(x, y) d x\right](1-\eta(y)) B_{0}(y) d y .
$$

It is easy to show this definition is independent of the choice of $\eta$ and each term on the right-hand side is well defined. Since our a priori assumptions mean that $B_{2}$ is smooth and has a smooth inverse, the decomposition of $H^{1}$ via smooth atoms and duality determine $T\left(B_{0}\right)$ as a BMO function.

We remark that, by duality, $T(F)$ can be identified as an element in $L^{\infty}(\mathbf{R}, \mathcal{M})$ if we can show that $|\langle G, T(F)\rangle| \leq C\|G\|_{L^{1}(\mathbf{R}, \mathcal{M})}$, and it certainly suffices to show that

$$
\sup _{x \in \mathbf{R}} \sup _{0<h<1}\left|\int_{\mathbf{R}} K_{h}(x, y) F(y) d y\right| \leq C .
$$

The version of the $T(B)$-Theorem that we will use is the following [10].

Theorem 4.6. Let $B_{1}$ and $B_{2}$ be two bounded functions from $\mathbf{R}$ to $\mathcal{M}$, such that their inverses exist and are bounded, all of these bounds being no more than some constant $C_{5}>0$. Let $T: B_{1} \mathcal{S} \rightarrow\left(B_{2} \mathcal{S}\right)^{\prime}$ be a singular integral operator such that $M_{B_{2}^{t}} T M_{B_{1}}$ is weakly bounded, with the constants $C$ appearing in (2.1), (2.2), (2.3) and (4.15) being no more than $C_{6}>0$, and $T\left(B_{1}\right)$ and $T^{t}\left(B_{2}\right)$ are in BMO with norms no more than $C_{7}>0$. Then $T$ admits a continuous extension onto $L^{2}(\mathbf{R}, \mathcal{M})$ with norm $\|T\|_{L^{2} \rightarrow L^{2}} \leq C\left(C_{5}+C_{6}+C_{7}\right)$.

With this result at hand, it is easy to see that Theorem 4.5 will be a consequence of the following lemmata.

Lemma 4.7. For each $\phi \in \Lambda^{\frac{k}{4}}\left(\varepsilon_{0}\right)$, the operator $M_{B_{0}^{t}} T M_{B_{1}}$ satisfies the weak boundedness property for any bounded $B_{0}$, the constant $C$ in (4.15) depending only on $\lambda, \Lambda, k$ and the bound on $B_{0}$. Also $T\left(B_{1}\right) \in L^{\infty}(\mathbf{R}, \mathcal{M})$, with $L^{\infty}$-norm bounded in terms of $\lambda, \Lambda$ and $k$.

Proof. We will first prove the weak boundedness of $M_{B_{0}^{t}} T M_{B_{1}}$. For any $i, j=1,2$ we choose

$$
\begin{aligned}
& u(X)=R^{-1} f_{i j}(X / R) h((t-\phi(x)) / R), \\
& v(X)=R^{-1} g_{i j}(x / R) h((t-\phi(x)) / R)
\end{aligned}
$$

and $b(X)=b_{0}^{i j}(\phi(x)) h(t)$ in (4.9), where $F=\left(f_{i j}\right)_{i j}$ and $G=\left(g_{i j}\right)_{i j}$ are normalised bumps, $B_{0}=\left(b_{0}^{i j}\right)_{i j}$ is a bounded function, and $h$ is a real-valued normalised bump centred at the origin and identically equal to one near the origin. We have

$$
\left|\int_{\Omega} \nabla u(Y) \cdot A^{t}(Y) \nabla \Gamma_{X}(Y) d Y\right| \leq\|\nabla u\|_{L^{\infty}\left(\mathbf{R}^{2}\right)}\left\|\nabla \Gamma_{X}\right\|_{L^{1}\left(B_{10 R}\right)} \leq C R^{-2+1},
$$

so

$$
\left|\int_{\partial \Omega_{h}}\left(\int_{\Omega} \nabla u(Y) \cdot A^{t}(Y) \nabla \Gamma_{X}(Y) d Y\right) b(X) v(X) d \sigma(X)\right| \leq C R^{-1} .
$$


Also

$$
\left|\int_{\partial \Omega_{h}} u(X) b(X) v(X) d X\right| \leq\|b\|_{L^{\infty}\left(\mathbf{R}^{2}\right)}\|u\|_{L^{2}(\partial \Omega)}\|v\|_{L^{2}(\partial \Omega)} \leq C R^{-1},
$$

so (4.9) allows us to conclude

$$
\left|\int_{\partial \Omega_{h}} \int_{\partial \Omega} u(Y) \nu(Y) \cdot A^{t}(Y) \nabla \Gamma_{X}(Y) b(X) v(X) d \sigma(Y) d \sigma(X)\right| \leq \frac{C}{R} .
$$

Now, if the supports of $u$ and $v$ are separated by a distance $R$, then we have

$$
\begin{aligned}
& \left|\int_{\partial \Omega_{h}} \int_{\partial \Omega}\left(\tau(Y) \cdot \nabla \Gamma_{X}(Y)\right) u(Y) b(X) v(X) d \sigma(Y) d \sigma(X)\right| \\
& =C R^{-1} \int_{\partial \Omega_{h}} \int_{\partial \Omega}|u(Y) b(X) v(X)| d \sigma(Y) d \sigma(X) \leq C R^{-1} .
\end{aligned}
$$

Otherwise, integration by parts shows us that

$$
\begin{aligned}
& \int_{\partial \Omega_{h}} \int_{\partial \Omega}\left(\tau(Y) \cdot \nabla \Gamma_{X}(Y)\right) u(Y) b(X) v(X) d \sigma(Y) d \sigma(X) \\
& =-\int_{\partial \Omega_{h}} \int_{\partial \Omega} \Gamma_{X}(Y)(\tau(Y) \cdot \nabla u(Y)) b(X) v(X) d \sigma(Y) d \sigma(X) .
\end{aligned}
$$

But since $u(Y) v(Y)=0$ for $|X-Y|>20 R,\|\nabla u\|_{L^{\infty}(\partial \Omega)} \leq C R^{-2}$ and $\|v\|_{L^{1}\left(\partial \Omega_{h}\right)} \leq$ $C$, we can rescale to obtain

$$
\begin{aligned}
& \left|\int_{\partial \Omega_{h}} \int_{\partial \Omega}\left(\tau(Y) \cdot \nabla \Gamma_{X}(Y)\right) u(Y) b(X) v(X) d \sigma(Y) d \sigma(X)\right| \\
& \leq C R^{-2} \sup _{X} \int_{\partial \Omega \cap B_{20 R}(X)}\left|\Gamma_{X}(Y)\right| d \sigma(Y) \\
& \leq C R^{-1} \int_{-R_{1}}^{R_{1}}|\log | y|| d y \leq C R^{-1} .
\end{aligned}
$$

The same argument as in the end of the proof of Lemma 4.3 shows us that the left-hand sides of (4.18), (4.19) and (4.20) are all we need to control in order to prove the weak boundedness of $M_{B_{2}^{t}} T M_{B_{1}}$.

To show that $T\left(B_{1}\right) \in L^{\infty}(\mathbf{R}, \mathcal{M})$ we will apply Green's second identity,

$$
\begin{gathered}
\varphi(X)-\int_{\Omega_{R}} \Gamma_{X}(Y) L \varphi(Y) d Y=\int_{\partial \Omega_{R}} \nu(Y) \cdot A^{t}(Y) \nabla \Gamma_{X}(Y) \varphi(Y) d \sigma(Y) \\
-\int_{\partial \Omega_{R}} \Gamma_{X}(Y) \nu(Y) \cdot A(Y) \nabla \varphi(Y) d \sigma(Y)
\end{gathered}
$$

to the domain $\Omega_{R}=\Omega \cap B_{R}(X)$ and the function $\varphi \equiv 1$. This leads to the equality

$$
\begin{aligned}
1= & \int_{\partial \Omega_{R}} \nu(Y) \cdot A^{t}(Y) \nabla \Gamma_{X}(Y) d \sigma(Y) \\
= & \int_{\partial \Omega \cap B_{R}(X)} \nu(Y) \cdot A^{t}(Y) \nabla \Gamma_{X}(Y) d \sigma(Y) \\
& +\int_{\Omega \cap \partial B_{R}(X)} \nu(Y) \cdot A^{t}(Y) \nabla \Gamma_{X}(Y) d \sigma(Y),
\end{aligned}
$$


but, using the estimate (4.1), we have

$$
\left|\int_{\Omega \cap \partial B_{R}(X)} \nu(Y) \cdot A^{t}(Y) \nabla \Gamma_{X}(Y) d \sigma(Y)\right| \leq C,
$$

so, letting $R \rightarrow \infty$ in the above, we obtain

$$
\left|\int_{\partial \Omega} \nu(Y) \cdot A^{t}(Y) \nabla \Gamma_{X}(Y) d \sigma(Y)\right| \leq C .
$$

We also have that, with $\Omega_{R}^{-}:=\Omega^{-} \cap B_{R}(X)$,

$$
\begin{aligned}
0= & \int_{\partial \Omega_{R}^{-}} \tau(Y) \cdot \nabla \Gamma_{X}(Y) d \sigma(Y) \\
= & \int_{\partial \Omega \cap B_{R}(X)} \tau(Y) \cdot \nabla \Gamma_{X}(Y) d \sigma(Y) \\
& +\int_{\Omega^{-} \cap \partial B_{R}(X)} \tau(Y) \cdot \nabla \Gamma_{X}(Y) d \sigma(Y),
\end{aligned}
$$

and in exactly the same fashion as before we can see that

$$
\left|\int_{\partial \Omega} \tau(Y) \cdot \nabla \Gamma_{X}(Y) d \sigma(Y)\right| \leq C
$$

So (as in the proof of Lemma 4.3), we have controlled all the terms necessary to prove the second statement of the lemma.

In order to apply Theorem 4.6 we need to show that $T^{t}\left(B_{2}\right) \in$ BMO. At this point the smallness of the Lipschitz constant will come in (specifically, in Lemma 4.11 and in the bound $C_{5}$ ). It will be necessary to first introduce a second singular integral operator $\widetilde{T}$ defined as

$$
\langle G, \widetilde{T}(F)\rangle=\lim _{h \searrow 0} \int_{\mathbf{R}^{2}} G(x)^{t} \widetilde{K}_{h}(x, y) F(y) d y d x,
$$

with $\widetilde{K}_{h}$ defined as follows. For the fundamental solution $\Gamma_{X}^{t}$ of $L^{t}=\operatorname{div} A^{t} \nabla$ and fixed $X$ let us define the conjugate $\widetilde{\Gamma}_{X}^{t}$ of $\Gamma_{X}^{t}$ to be

$$
\widetilde{\Gamma}_{X}^{t}(Y)=\int_{\gamma\left(Y_{0}, Y\right)} \nu(Z) \cdot A(Z) \nabla \Gamma_{X}^{t}(Z) d l(Z)
$$

on the complement of the set $\{Y=(y, s) \mid s \geq t, y=x\}$. Here $\gamma\left(Y_{0}, Y\right)$ is a path from a fixed point $Y_{0}$ to $Y$ parametrised by arc length via the function $t \mapsto$ $\left(l_{1}(t), l_{2}(t)\right)$ and remaining in the complement of $\{Z=(z, r) \mid r \geq t, z=x\}$. Also $\nu(Z)=\left(l_{2}^{\prime}(t),-l_{1}^{\prime}(t)\right)$ is the unit normal to $\gamma\left(Y_{0}, Y\right)$ at $Z=\left(l_{1}(t), l_{2}(t)\right)$ and $d l$ is arc length. It is easy to see $\widetilde{\Gamma}_{X}^{t}(Y)$ solves the system

$$
A(Y) \nabla \Gamma_{X}^{t}(Y)=\left(\begin{array}{cc}
0 & 1 \\
-1 & 0
\end{array}\right) \nabla \widetilde{\Gamma}_{X}^{t}(Y)
$$

The function $Y \mapsto \widetilde{\Gamma}_{X}^{t}(Y)$ is well defined up to a constant (which depends on the choice of $Y_{0}$ ). Set

$$
\left(\begin{array}{c}
\widetilde{k}_{1, h}(x, y) \\
\widetilde{k}_{2, h}(x, y)
\end{array}\right)=\nabla_{X} \widetilde{\Gamma}_{(x, \phi(x)+h)}^{t}(y, \phi(y))
$$


and define

$$
\widetilde{K}_{h}=\left(\begin{array}{cc}
\widetilde{k}_{1, h} & \widetilde{k}_{2, h} \\
\widetilde{k}_{1, h} & \widetilde{k}_{2, h}
\end{array}\right) .
$$

As before we write $\widetilde{K}_{0}=\widetilde{K}$.

Lemma 4.8. For each $\phi \in \Lambda^{\frac{k}{4}}\left(\varepsilon_{0}\right)$, the operator $\widetilde{T}$ defined above is a singular integral operator associated to the Calderón-Zygmund kernel $\widetilde{K}$. More precisely, $\widetilde{K}$ satisfies estimates (2.1), (2.2) and (2.3) with the constant $C=C_{8}$ depending only on $\lambda, \Lambda$ and $k$, and $\widetilde{T}: B_{3} \mathcal{S} \rightarrow\left(B_{1} \mathcal{S}\right)^{\prime}$ is a continuous linear operator with representation (2.4).

Proof. The estimates (2.1), (2.2) and (2.3) may be easily obtained from the estimates (4.1), (4.2) and (4.3) with $\nabla_{X} \widetilde{\Gamma}_{X}^{t}$ replacing $\nabla \Gamma_{X}$, so it suffices to show the latter. We also observe that, since $\phi \in \Lambda^{\frac{k}{4}}\left(\varepsilon_{0}\right)$, we only need to prove (4.1), (4.2) and (4.3) for $X=(x, t) \in\{Z=(z, r)|| r-s|\leq(k / 2)| z-y \mid\}$, where $Y=(y, s)$. Since $X \mapsto \widetilde{\Gamma}_{X}^{t}(Y)$ is a solution to the non-divergence form equation $L^{t} u=0$ and $Y \mapsto \widetilde{\Gamma}_{X}^{t}(Y)$ is a solution to the divergence form equation $\operatorname{div} \widetilde{A} \nabla u=0$ when $X \neq Y$, we may repeat the proof of Lemma 4.1 once we have shown (4.1) for $\nabla_{X} \widetilde{\Gamma}_{X}^{t}$. From (4.22) we have

$$
\nabla_{X} \widetilde{\Gamma}_{X}^{t}(Y)=\int_{\gamma\left(Y_{0}, Y\right)} \nabla_{X}\left(\nu(Z) \cdot A(Z) \nabla \Gamma_{X}^{t}(Z)\right) d l(Z) .
$$

Now, with the a priori smoothness assumption, the integrand here has size $C /|X-Z|^{2}$ for $Z$ away from $X$, so

$$
\int_{\gamma\left(Y_{1}, Y_{2}\right)} \nabla_{X}\left(\nu(Z) \cdot A(Z) \nabla \Gamma_{X}^{t}(Z)\right) d l(Z) \rightarrow 0
$$

as $\min \left(\left|Y_{1}-X\right|,\left|Y_{2}-X\right|\right) \rightarrow \infty$, which says we can write

$$
\nabla_{X} \widetilde{\Gamma}_{X}^{t}(Y)=\int_{\gamma_{\theta}(Y)} \nabla_{X}\left(\nu(Z) \cdot A(Z) \nabla \Gamma_{X}^{t}(Z)\right) d l(Z),
$$

where $\gamma_{\theta}(Y)$ is a ray from infinity to $Y$ approaching $Y$ at an angle $\theta$ such that $\arctan (k / 2)-\pi \leq \theta \leq-\arctan (k / 2)$. Averaging over $\theta$ we obtain

$$
\begin{aligned}
& \nabla_{X} \widetilde{\Gamma}_{X}^{t}(Y)=f_{\arctan k-\pi}^{-\arctan k} \int_{\gamma_{\theta}(Y)} \nabla_{X}\left(\nu(Z) \cdot A(Z) \nabla \Gamma_{X}^{t}(Z)\right) d l(Z) d \theta \\
& =C \int_{\Gamma_{1}^{-}(Y)} \nabla_{X}\left(\nu(Z) \cdot A(Z) \nabla \Gamma_{X}^{t}(Z)\right) \frac{d Z}{|Z-Y|} \\
& =C \int_{\Gamma_{1}^{-}(Y) \cap B_{|X-Y| / 8}(Y)} \nabla_{X}\left(\nu(Z) \cdot A(Z) \nabla \Gamma_{X}^{t}(Z)\right) \frac{d Z}{|Z-Y|} \\
& +C \sum_{n=-2}^{\infty} \int_{\Gamma_{1}^{-}(Y) \cap\left(B_{2^{n}|X-Y|}(Y) \backslash B_{2^{n-1}|X-Y|}(Y)\right)} \nabla_{X}\left(\nu(Z) \cdot A(Z) \nabla \Gamma_{X}^{t}(Z)\right) \frac{d Z}{|Z-Y|} \\
& =Q_{1}+Q_{2},
\end{aligned}
$$

where $\Gamma_{1}^{-}(Y)=\left\{Z \in \mathbf{R}^{2}|s-r>k| z-y \mid\right\}$. Define also $\Gamma_{2}^{-}(Y)=\left\{Z \in \mathbf{R}^{2} \mid s-r>\right.$ $(3 k / 4)|z-y|\}$ and $\Gamma_{3}^{-}(Y)=\left\{Z \in \mathbf{R}^{2}|s-r>(k / 2)| z-y \mid\right\}$. Observe that since 
$X \notin \Gamma_{3}^{-}(Y)$ and $Z \in \Gamma_{1}^{-}(Y)$ we have $|X-Z| \geq C(|X-Y|+|Z-Y|)$. Thus using Hölder's inequality, Lemma 2.1 and (4.1), we can estimate

$$
\begin{aligned}
& Q_{2} \leq C \sum_{n=-2}^{\infty} \int_{\Gamma_{1}^{-}(Y) \cap\left(B_{2^{n}|X-Y|}(Y) \backslash B_{2^{n-1}|X-Y|}(Y)\right)}\left|\nabla_{X} \nabla \Gamma_{X}^{t}(Z)\right| \frac{d Z}{|Z-Y|} \\
& \leq C \sum_{n=-2}^{\infty}\left(\int_{\Gamma_{1}^{-}(Y) \cap\left(B_{2^{n}|X-Y|}(Y) \backslash B_{2^{n-1}|X-Y|}(Y)\right)}\left|\nabla_{X} \nabla \Gamma_{X}^{t}(Z)\right|^{2} d Z\right)^{\frac{1}{2}} \\
& \leq C \sum_{n=-2}^{\infty} \frac{2^{-n}}{|X-Y|}\left(\int_{\Gamma_{2}^{-}(Y) \cap\left(B_{2^{n}|X-Y|}(Y) \backslash B_{2^{n-1}|X-Y|}(Y)\right)}\left|\nabla_{X} \Gamma_{X}^{t}(Z)\right|^{2} d Z\right)^{\frac{1}{2}} \\
& \leq C \sum_{n=-2}^{\infty} \frac{2^{-n}}{|X-Y|}\left(\int_{\Gamma_{2}^{-}(Y) \cap\left(B_{2^{n}|X-Y|}(Y) \backslash B_{2^{n-1}|X-Y|}(Y)\right)} \frac{1}{|X-Z|^{2}} d Z\right)^{\frac{1}{2}} \\
& \leq C \sum_{n=-2}^{\infty} \frac{2^{-n}}{|X-Y|}\left(\int_{\Gamma_{2}^{-}(Y) \cap\left(B_{2^{n}|X-Y|}(Y) \backslash B_{2^{n-1}|X-Y|}(Y)\right)} \frac{1}{\left(2^{n-1}|X-Y|\right)^{2}} d Z\right)^{\frac{1}{2}} \\
& \leq C \sum_{n=-2}^{\infty} \frac{2^{-n}}{|X-Y|} \leq \frac{C}{|X-Y|} \text {. }
\end{aligned}
$$

Also, using Hölder's inequality, Lemma 2.3 and Lemma 2.1, we have, for some $p>2$,

$$
\begin{aligned}
& Q_{1} \leq C \int_{B_{|X-Y| / 8}(Y)}\left|\nabla_{X} \nabla \Gamma_{X}^{t}(Z)\right| \frac{d Z}{|Z-Y|} \\
& \leq C\left(\int_{B_{|X-Y| / 8}(Y)} \frac{1}{|Z-Y|^{p^{\prime}}} d Z\right)^{\frac{1}{p^{\prime}}}\left(\int_{B_{|X-Y| / 8}(Y)}\left|\nabla_{X} \nabla \Gamma_{X}^{t}(Z)\right|^{p} d Z\right)^{\frac{1}{p}} \\
& =C|X-Y|^{\frac{2-p^{\prime}}{p}}\left(\int_{B_{|X-Y| / 8}(Y)}\left|\nabla_{X} \nabla \Gamma_{X}^{t}(Z)\right|^{p} d Z\right)^{\frac{1}{p}} \\
& \leq C\left(\int_{B_{|X-Y| / 4}(Y)}\left|\nabla_{X} \nabla \Gamma_{X}^{t}(Z)\right|^{2} d Z\right)^{\frac{1}{2}} \\
& \leq \frac{C}{|X-Y|}\left(\int_{B_{|X-Y| / 2}(Y)}\left|\nabla_{X} \Gamma_{X}^{t}(Z)\right|^{2} d Z\right)^{\frac{1}{2}} \leq \frac{C}{|X-Y|} .
\end{aligned}
$$

This proves (4.1) for $\nabla_{X} \widetilde{\Gamma}_{X}^{t}$, and the other two estimates (4.2) and (4.3) follow as before.

To prove the continuity of $\widetilde{T}: B_{3} \mathcal{S} \rightarrow\left(B_{1} \mathcal{S}\right)^{\prime}$ we again fix $f, g \in \mathcal{S}(\mathbf{R}, \mathbf{R}), h \in$ $C_{0}^{\infty}(\mathbf{R}, \mathbf{R})$ with $h$ positive and equal to one near zero. Let $\delta_{0}: \Omega^{-} \rightarrow \mathbf{R}$ be the Dahlberg-Kenig-Stein adapted distance function introduced in [7] (see also [8]). Define $u, v: \Omega \rightarrow \mathbf{R}$ by

$$
u(X)=f(x) h\left(\delta_{0}(x, t)\right) \text { and } v(X)=g(x) h(t-\phi(x)) .
$$


Define $\mu: \mathbf{R}^{2} \rightarrow \mathbf{R}$ by $\mu(X)=\left(1, \alpha_{0}\right) \cdot(x, t)$. Then $\nabla_{X} \mu(X-Z)=\left(1, \alpha_{0}\right)=\kappa$ for all $Z \in \mathbf{R}^{2}$. For $F: \Omega^{-} \rightarrow \mathbf{R}$ we define (see [4, 24]) the quantities

$$
\mathfrak{T}(F)(Q):=\sup _{\Delta_{r} \ni Q}\left(\frac{1}{\left|\Delta_{r}\right|} \int_{B_{r}(Q)}|F(Y)|^{2} \frac{d Y}{\delta(Y)}\right)^{\frac{1}{2}}
$$

where $\Delta_{r}=B_{r}\left(Q^{\prime}\right) \cap \partial \Omega$ and $Q^{\prime} \in \partial \Omega$, and

$$
\mathfrak{S}(F)(Q):=\left(\int_{\Gamma^{-}(Q)}|F(Y)|^{2} \frac{d Y}{\delta(Y)^{2}}\right)^{\frac{1}{2}} .
$$

We have that

$$
\int_{\Omega^{-}}|F(Y) G(Y)| \frac{d Y}{\delta(Y)} \leq C \int_{\partial \Omega} \mathfrak{T}(F)(Q) \mathfrak{S}(G)(Q) d \sigma(Q)
$$

It is well known that $\left\|\nabla \delta_{0}\right\|_{L^{\infty}(\Omega, \mathbf{R})}$ and $\left\|\mathfrak{T}\left(\delta_{0} \partial^{2} \delta_{0}\right)\right\|_{L^{\infty}(\partial \Omega, \mathbf{R})}$ are bounded in terms of the Lipschitz constant, where $\partial^{2}$ denotes any second-order partial derivative, and $\delta \simeq \delta_{0}$.

We apply Green's second identity to the function $\mu(\cdot-Z) u(\cdot)$ and the fundamental solution $\Gamma_{X}^{t}$ in the domain $\Omega^{-}$. We obtain

$$
\begin{aligned}
& \mu(X-Z) u(X)-\int_{\Omega^{-}} \Gamma_{X}^{t}(Y) L^{t}(\mu(\cdot-Z) u(\cdot))(Y) d Y \\
& =\int_{\partial \Omega} \nu(Y) \cdot A(Y) \nabla \Gamma_{X}^{t}(Y) \mu(Y) u(Y) d \sigma(Y) \\
& \quad-\int_{\partial \Omega} \Gamma_{X}^{t}(Y) \nu(Y) \cdot A^{t}(Y)(u(Y) \kappa+\mu(Y-Z) \nabla u(Y)) d \sigma(Y) .
\end{aligned}
$$

Using (4.23) and integration by parts in the first term on the right-hand side, we see the above is equal to

$$
\begin{aligned}
& \mu(X-Z) u(X)-\int_{\Omega^{-}} \Gamma_{X}^{t}(Y) L^{t}(\mu(\cdot-Z) u(\cdot)(Y) d Y \\
& =-\int_{\partial \Omega} \widetilde{\Gamma}_{X}^{t}(Y) \tau(Y) \cdot(u(Y) \kappa+\mu(Y-Z) \nabla u(Y)) d \sigma(Y) \\
& \quad-\int_{\partial \Omega} \Gamma_{X}^{t}(Y) \nu(Y) \cdot A^{t}(Y)(u(Y) \kappa+\mu(Y-Z) \nabla u(Y)) d \sigma(Y) .
\end{aligned}
$$

The above integration by parts is the reason for introducing $\widetilde{\Gamma}_{X}^{t}$; see also (4.28) and (4.29). Taking the gradient in $X$, multiplying by $v \mathbf{b}$, where $\mathbf{b}: \partial \Omega_{h} \rightarrow \mathbf{R}^{2}$ is a 
bounded function, setting $Z=X$ and integrating in $X$, we find

$$
\begin{aligned}
& \int_{\partial \Omega_{h}} u(X) v(X) \mathbf{b}(X) \cdot \kappa d \sigma(X) \\
& \quad-\int_{\partial \Omega_{h}} v(X) \mathbf{b}(X) \cdot\left(\int_{\Omega^{-}} \nabla_{X} \Gamma_{X}^{t}(Y) L^{t}(\mu(\cdot-X) u(\cdot))(Y) d Y\right) d \sigma(X) \\
& =-\int_{\partial \Omega_{h}} v(X) \mathbf{b}(X) \cdot \int_{\partial \Omega} \nabla_{X} \widetilde{\Gamma}_{X}^{t}(Y) \\
& \quad \times\{\tau(Y) \cdot(u(Y) \kappa+\mu(Y-X) \nabla u(Y))\} d \sigma(Y) d \sigma(X) \\
& \quad-\int_{\partial \Omega_{h}} v(X) \mathbf{b}(X) \cdot \int_{\partial \Omega} \nabla_{X} \Gamma_{X}^{t}(Y) \\
& \quad \times\left\{\nu(Y) \cdot A^{t}(Y)(u(Y) \kappa+\mu(Y-X) \nabla u(Y))\right\} d \sigma(Y) d \sigma(X) .
\end{aligned}
$$

The first term on the right-hand side can be expanded:

$$
\begin{aligned}
& \int_{\partial \Omega_{h}} v(X) \mathbf{b}(X) \cdot \int_{\partial \Omega} \nabla_{X} \widetilde{\Gamma}_{X}^{t}(Y) u(Y)(\tau(Y) \cdot \kappa) d \sigma(Y) d \sigma(X) \\
& \left.+\int_{\partial \Omega_{h}} v(X) \mathbf{b}(X) \cdot \int_{\partial \Omega} \nabla_{X} \widetilde{\Gamma}_{X}^{t}(Y) \mu(Y-X) \tau(Y) \cdot \nabla u(Y)\right\} d \sigma(Y) d \sigma(X) .
\end{aligned}
$$

The first term in this expression is exactly what we need to control to show the continuity of $\widetilde{T}: B_{3} \mathcal{S} \rightarrow\left(B_{1} \mathcal{S}\right)^{\prime}$ when $\mathbf{b}$ is chosen to be either one of the columns of $B_{1}$. Moreover, the second term on the right-hand side of (4.25) has already been seen to be controllable by a finite linear combination of $[f]_{N}^{\beta}[g]_{N}^{\beta}$, since the proof of Lemma 4.3 shows that $\left(T_{-}\right)^{t}: B_{0} \mathcal{S} \rightarrow\left(B_{1} \mathcal{S}\right)^{\prime}$ is continuous for any bounded $B_{0}$, where $T_{-}$is the operator defined as in (4.8), but with the limit as $h \nearrow 0$. To control the second term on the left-hand side, we have

$$
\left|L^{t}(\mu(\cdot-X) u(\cdot))\right| \leq\left|\mu(\cdot-X) L^{t}(u)\right|+C|\nabla u|,
$$

so

$$
\begin{aligned}
& \int_{\Omega^{-}} \nabla_{X} \Gamma_{X}^{t}(Y) L^{t}(\mu(\cdot-X) u(\cdot))(Y) d Y \\
& \leq C \int_{\Omega^{-}}\left|L^{t}(u)(Y)\right| d Y+C \int_{\Omega^{-}} \frac{|\nabla u(Y)|}{|X-Y|} d Y .
\end{aligned}
$$

The second term is easy to control by $C\left([f]_{2}^{1}+[f]_{2}^{0}\right)$ and so its contribution to the second term on the left-hand side of (4.25) is also controlled. The first term here requires a little more work. Let us consider just the first term $\partial_{11} u$ of $L^{t}(u)$, as the same analysis can be used on the other terms. We have

$$
\begin{aligned}
\partial_{11} u(X)= & f^{\prime \prime}(x) h\left(\delta_{0}(x, t)\right)+f^{\prime}(x) h^{\prime}\left(\delta_{0}(x, t)\right) \partial_{1} \delta_{0}(x, t) \\
& +f(x) h^{\prime \prime}\left(\delta_{0}(x, t)\right)\left(\partial_{1} \delta_{0}(x, t)\right)^{2}+f(x) h^{\prime}\left(\delta_{0}(x, t)\right)\left(\partial_{11} \delta_{0}(x, t)\right),
\end{aligned}
$$

so the contribution to the double integral from the first three terms is easily bounded by $C\left([f]_{2}^{2}+[f]_{2}^{1}+[f]_{2}^{0}\right)$. The last term is controlled using (4.24):

$$
\int_{\Omega^{-}}\left|f(y) h^{\prime}\left(\delta_{0}(y, s)\right)\left(\partial_{11} \delta_{0}(y, s)\right)\right| d Y \leq C \int_{\partial \Omega} \mathfrak{T}\left(f h^{\prime}\left(\delta_{0}\right)\right) \mathfrak{S}\left(\delta_{0} \partial_{11} \delta_{0}\right) d \sigma \leq C[f]_{2}^{0},
$$

since $\left\|\mathfrak{S}\left(\delta_{0} \partial_{11} \delta_{0}\right)\right\|_{L^{\infty}(\partial \Omega, \mathbf{R})} \leq C$ and it is clear from the support properties of $h^{\prime}(\delta)$ that $\left\|\mathfrak{S}\left(f h^{\prime}\left(\delta_{0}\right)\right)\right\|_{L^{1}(\partial \Omega, \mathbf{R})} \leq C[f]_{2}^{0}$. Thus, the second term on the left-hand side of (4.25) is also controlled. The remaining terms in (4.25) and (4.26) may 
be controlled in terms of a finite linear combination of $[f]_{N}^{\beta}[g]_{N}^{\beta}$ directly, as was done in the proof of Lemma 4.3. We remark that under our a priori smoothness assumptions the limit (4.21) can be seen to exist, again as was done in the proof of Lemma 4.3 .

Remark 4.9. Note that, in fact, we have estimate (4.1) for $\nabla_{X} \widetilde{\Gamma}_{X}^{t}(Y)$ when $Y \notin$ $\{Z|r-t>(k / 2)| z-x \mid)\}$, that is, away from a cone with axis being the ray on which $\widetilde{\Gamma}_{X}^{t}$ is not defined. This will be used in Lemma 4.10.

Lemma 4.10. For each $\phi \in \Lambda^{\frac{k}{4}}\left(\varepsilon_{0}\right)$, the operator $M_{B_{1}^{t}} \widetilde{T} M_{B_{3}}$ satisfies the weak boundedness property and $\widetilde{T}^{t}\left(B_{1}\right) \in L^{\infty}(\mathbf{R}, \mathcal{M})$, with bounds depending only on $\lambda$, $\Lambda$ and $k$.

Proof. We can prove the weak boundedness by using (4.25) with $u$ and $v$ replaced by

$$
\begin{aligned}
& u(X)=R^{-1} f_{i j}(X / R) h\left(\delta_{0}(x, t) / R\right) \text { and } \\
& v(X)=R^{-1} g_{i j}(x / R) h((t-\phi(x)) / R),
\end{aligned}
$$

with $f_{i j}, g_{i j}$ and $h$ as in (4.17), and $\mathbf{b}$ again being either one of the columns of $B_{1}$. The estimate we require is that

$$
\left|\int_{\partial \Omega_{h}} v(X) \mathbf{b}(X) \cdot \int_{\partial \Omega} \nabla_{X} \widetilde{\Gamma}_{X}^{t}(Y) u(Y)(\tau(Y) \cdot \kappa) d \sigma(Y) d \sigma(X)\right| \leq \frac{C}{R},
$$

which is part of the first term on the right-hand side of (4.25). The second term on the right-hand side of (4.25) is controlled in the necessary manner because $M_{B_{1}^{t}}\left(T_{-}\right)^{t} M_{B_{0}}$ satisfies the weak boundedness property for any bounded $B_{0}$ (as the proof of Lemma 4.7 shows). The second term on the left-hand side of (4.25) is controlled in the same manner as in the proof of Lemma 4.8, and the remaining terms can be estimated directly, leaving us with the required estimate.

To show that $\widetilde{T}^{t}\left(B_{1}\right) \in L^{\infty}(\mathbf{R}, \mathcal{M})$, we will apply Green's second identity,

$$
\begin{gathered}
-\int_{\Omega_{h, R}} \widetilde{\Gamma}_{X}(Y) L \varphi(X) d X=\int_{\partial \Omega_{h, R}} \nu(X) \cdot A^{t}(X) \nabla_{X} \widetilde{\Gamma}_{X}(Y) \varphi(X) d \sigma(X) \\
-\int_{\partial \Omega_{h, R}} \widetilde{\Gamma}_{X}(Y) \nu(X) \cdot A(X) \nabla \varphi(X) d \sigma(X)
\end{gathered}
$$

to the domain $\Omega_{h, R}:=\{X=(x, t) \mid t>\phi(x)+h\} \cap B_{R}(Y)$ and the functions $X \mapsto \widetilde{\Gamma}_{X}^{t}(Y)$ and $\varphi \equiv 1$. This leads to the equality

$$
\begin{aligned}
0= & \int_{\partial \Omega_{h, R}} \nu(X) \cdot A^{t}(X) \nabla_{X} \widetilde{\Gamma}_{X}(Y) d \sigma(X) \\
= & \int_{\partial \Omega_{h} \cap B_{R}(Y)} \nu(X) \cdot A^{t}(X) \nabla_{X} \widetilde{\Gamma}_{X}(Y) d \sigma(X) \\
& +\int_{\Omega_{h} \cap \partial B_{R}(Y)} \nu(X) \cdot A^{t}(X) \nabla_{X} \widetilde{\Gamma}_{X}(Y) d \sigma(X)
\end{aligned}
$$

but, by Remark 4.9. we have

$$
\left|\int_{\Omega_{h} \cap \partial B_{R}(X)} \nu(X) \cdot A^{t}(X) \nabla_{X} \widetilde{\Gamma}_{X}(Y) d \sigma(X)\right| \leq C
$$


so, letting $R \rightarrow \infty$ in the above, we obtain

$$
\left|\int_{\partial \Omega} \nu(X) \cdot A^{t}(X) \nabla_{X} \widetilde{\Gamma}_{X}(Y) d \sigma(X)\right| \leq C
$$

The bound

$$
\left|\int_{\partial \Omega_{h}} \tau(X) \cdot \nabla_{X} \widetilde{\Gamma}_{X}^{t}(Y) d \sigma(X)\right| \leq C
$$

also follows via the method used to prove Lemma 4.7 on $\Omega_{h, R}$. These two estimates bound all the components of $\widetilde{T}^{t}\left(B_{1}\right)$.

Lemma 4.11. For each $\phi \in \Lambda^{\frac{k}{4}}\left(\varepsilon_{0}\right)$, we have the inequalities $\left\|\widetilde{T}\left(B_{3}\right)\right\|_{B M O} \leq C+$ $C\left\|T^{t}\left(B_{2}\right)\right\|_{B M O}$ and $\left\|T^{t}\left(B_{2}\right)\right\|_{B M O} \leq C+C \varepsilon_{0}\left\|\widetilde{T}\left(B_{3}\right)\right\|_{B M O}$, with the constants $C$ depending only on $\lambda, \Lambda$ and $k$.

Proof. To prove this lemma we will use the $H^{1}$-BMO duality and the smooth atomic decomposition of $H^{1}$ (for a discussion of this see, for example, [14]). For each $H^{1}$ atom $A_{0} \in B_{1} \mathcal{S}$, with support in a ball $B_{R}\left(x_{0}\right)$ of radius $R$, we choose an $\eta: \mathbf{R} \rightarrow \mathbf{R}$ with support in $B_{4 R}\left(x_{0}\right)$ and identically equal to one on $B_{2 R}\left(x_{0}\right)$ such that $\left|\eta^{\prime}\right| \leq C / R$ and $\left|\eta^{\prime \prime}\right| \leq C / R^{2}$. Recall that with our a priori smoothness assumptions such an atom $A_{0}$ is in fact an arbitrary smooth atom. To prove the first inequality we use definition (4.16) to compute $\left\langle A_{0}, \widetilde{T}\left(B_{3}\right)\right\rangle$. The second term on the right-hand side of (4.16) is controlled by a multiple of $\left\|B_{3}\right\|_{\infty}$, and so we only need to estimate $\left\langle A_{0}, \widetilde{T}\left(\eta B_{3}\right)\right\rangle$. To that end, we define $\varphi: \mathbf{R}^{2} \rightarrow \mathbf{R}$ by

$$
\varphi(X)=\eta(x) \eta\left(\delta_{0}(x, t)\right),
$$

where we recall $X=(x, t)$ and $\delta_{0}$ is the adpated distance function, and repeat the calculation that yielded (4.25) with $\mu(\cdot-Z) \varphi(\cdot)$ and $\tilde{a}$, where $\tilde{a}(x, \phi(x)+h)=$ $a(x) /\left(1+\phi^{\prime}(x)\right)^{\frac{1}{2}}$ and $a$ is any matrix element of $A_{0}$. This gives

$$
\begin{aligned}
& \int_{\partial \Omega_{h}} \tilde{a}(X) \varphi(X) \kappa d \sigma(X) \\
& \quad+\int_{\partial \Omega_{h}} \tilde{a}(X)\left(\int_{\Omega^{-}} \nabla_{X} \Gamma_{X}^{t}(Y) L^{t}(\mu(\cdot-X) \varphi(\cdot))(Y) d Y\right) d \sigma(X) \\
& =\int_{\partial \Omega_{h}} \tilde{a}(X) \int_{\partial \Omega} \nabla_{X} \widetilde{\Gamma}_{X}^{t}(Y) \\
& \quad \times\{\tau(Y) \cdot(\varphi(Y) \kappa+\mu(Y-X) \nabla \varphi(Y))\} d \sigma(Y) d \sigma(X) \\
& \quad+\int_{\partial \Omega_{h}} \tilde{a}(X) \int_{\partial \Omega} \nabla_{X} \Gamma_{X}^{t}(Y) \\
& \quad \times\left\{\nu(Y) \cdot A^{t}(Y)(\varphi(Y) \kappa+\mu(Y-X) \nabla \varphi(Y))\right\} d \sigma(Y) d \sigma(X) .
\end{aligned}
$$

The first term on the left-hand side of (4.28) is easy to control. Indeed,

$$
\begin{aligned}
& \int_{\partial \Omega_{h}} \tilde{a}(X) \varphi(X) \kappa d \sigma(X) \\
& \leq C\|a\|_{L^{2}(\mathbf{R})}\|\varphi \kappa\|_{L^{2}\left(\partial \Omega_{h}\right)} \leq C R^{\frac{1}{2}} / R^{\frac{1}{2}} .
\end{aligned}
$$

To control the second term on the left-hand side of (4.28) we expand

$$
L^{t}(\mu \varphi)=\varphi L^{t} \mu+\mu L^{t} \varphi+2 \partial_{1} \varphi \partial_{1} \mu+b\left(\partial_{1} \varphi \partial_{2} \mu+\partial_{2} \varphi \partial_{1} \mu\right)+2 c \partial_{2} \varphi \partial_{2} \mu .
$$


The first term here is zero and the other terms except for $\mu L^{t} \varphi$ are of size $C / R$. Now

$$
\begin{aligned}
\partial_{11} \varphi(x, t)= & \eta^{\prime \prime}(x) \eta\left(\delta_{0}(x, t)\right)+2 \eta^{\prime}(x) \eta^{\prime}\left(\delta_{0}(x, t)\right) \partial_{1} \delta_{0}(x, t) \\
& +\eta(x) \eta^{\prime \prime}\left(\delta_{0}(x, t)\right)\left(\partial_{1} \delta_{0}(x, t)\right)^{2}+\eta(x) \eta^{\prime}\left(\delta_{0}(x, t)\right) \partial_{11} \delta_{0}(x, t),
\end{aligned}
$$

so each term here is of size $C / R^{2}$ except for the term where two derivatives fall on $\delta_{0}$. Since the same is true for the other second-order derivatives of $\varphi$ we can say $\mu L^{t} \varphi$ is a sum of terms of size $C / R$ plus terms involving the second-order derivatives of $\delta_{0}$. We conclude that

$$
L^{t}(\mu \varphi)=\eta \eta^{\prime}\left(\delta_{0}\right) \sum_{i, j=1,2} c_{i j} \partial_{i j} \delta_{0}+Q,
$$

where $Q$ is a sum of terms of size $C / R$ and $c_{i j}(i, j=1,2)$ are functions of size $C|Y-X|$. So for $0<h<\min \{1, R / 2\}$,

$$
\left|\int_{\Omega^{-} \cap\{R<\delta(Y)<4 R\}} \nabla_{X} \Gamma_{X}^{t}(Y) Q(Y) d Y\right| \leq \frac{C R^{2}}{R R} \leq C .
$$

Moreover, when $0<\delta(Y)<R$ on $\Omega^{-}$the supports of $\tilde{a}$ and $\nabla \varphi$ are disjoint and separated by a distance $C R$, so it follows that the same is true of $\tilde{a}$ and $Q$; thus $\left|\nabla_{X} \Gamma_{X}^{t}(Y)\right| \leq C / R$, so once again

$$
\left|\int_{\Omega^{-} \cap\{0<\delta(Y)<R\}} \nabla_{X} \Gamma_{X}^{t}(Y) Q(Y) d Y\right| \leq \frac{C R^{2}}{R R} \leq C .
$$

Since the integrand in the integral over $\Omega^{-}$in (4.28) is supported on $4 B$, the contribution from $Q$ to the second term on the left-hand side of (4.28) is now controlled. The contribution from $\eta \eta^{\prime}\left(\delta_{0}\right) \sum c_{i j} \partial_{i j} \delta_{0}$ is again controlled using (4.24) as in the proof of Lemma 4.8 .

We will now consider the first term on the right-hand side of (4.28):

$$
\begin{aligned}
& \int_{\partial \Omega_{h}} \tilde{a}(X) \int_{\partial \Omega} \nabla_{X} \widetilde{\Gamma}_{X}^{t}(Y) \tau(Y) \cdot \kappa \varphi(Y) d \sigma(Y) d \sigma(X) \\
& +\int_{\partial \Omega_{h}} \tilde{a}(X) \int_{\partial \Omega} \nabla_{X} \widetilde{\Gamma}_{X}^{t}(Y) \tau(Y) \cdot \nabla \varphi(Y) \mu(Y-X) d \sigma(Y) d \sigma(X) .
\end{aligned}
$$

The first term is a component of $\left\langle A_{0}, \widetilde{T}\left(\eta B_{3}\right)\right\rangle$ and the second is easily seen to be bounded by a constant using (4.1). In exactly the same manner, the second term on the right-hand side of (4.28) is bounded by $\left|\left\langle A_{0}, T^{t}\left(\eta B_{0}\right)\right\rangle\right|+C$, for some bounded $B_{0}$. All this yields the estimate

$$
\left|\left\langle A_{0}, \widetilde{T}\left(\eta B_{3}\right)\right\rangle\right| \leq C+C\left|\left\langle A_{0}, T^{t}\left(\eta B_{0}\right)\right\rangle\right|,
$$

from which duality, standard Calderón-Zygmund theory, Theorem 4.6 and Lemma 4.7 allow us to conclude

$$
\left\|\widetilde{T}\left(\eta B_{3}\right)\right\|_{\mathrm{BMO}} \leq C+C\left\|T^{t}\left(B_{2}\right)\right\|_{\text {вмо }} .
$$

The first inequality of the lemma follows from this.

The second inequality follows by the same analysis, replacing $\mu(\cdot-Z)$ by $\xi(\cdot-Z)$ in the calculation that gave (4.28), where $\xi(X)=\left(-\alpha_{0}, 1\right) \cdot(x, t)$. The first term 
on the right-hand side of the equation which replaces (4.28) is

$$
\begin{aligned}
& \int_{\partial \Omega_{h}} \tilde{a}(X) \int_{\partial \Omega} \nabla_{X} \widetilde{\Gamma}_{X}^{t}(Y)\left(\tau(Y) \cdot \kappa^{\perp}\right) \varphi(Y) d \sigma(Y) d \sigma(X) \\
& +\int_{\partial \Omega_{h}} \tilde{a}(X) \int_{\partial \Omega} \nabla_{X} \widetilde{\Gamma}_{X}^{t}(Y)(\tau(Y) \cdot \nabla \varphi(Y)) \xi(Y-X) d \sigma(Y) d \sigma(X) .
\end{aligned}
$$

We have that $\left(1+\left(\phi^{\prime}\right)^{2}\right)^{\frac{1}{2}} \tau \cdot \kappa^{\perp}=\phi^{\prime}-\alpha_{0}$, which is bounded in absolute value by $\varepsilon_{0}$, so the first term in (4.29) is a component of $\left\langle A_{0}, \widetilde{T}\left(B_{\varepsilon_{0}}\right)\right\rangle$, where $B_{\varepsilon_{0}}$ is a function bounded by $C \varepsilon_{0}$. The second term in (4.29) is bounded by a constant as before. The second term on the right-hand side of the equation which replaces (4.28) is

$$
\begin{aligned}
& \int_{\partial \Omega_{h}} \tilde{a}(X) \int_{\partial \Omega} \nabla_{X} \Gamma_{X}^{t}(Y)\left(\nu(Y) \cdot A^{t}(Y) \kappa^{\perp}\right) \varphi(Y) d \sigma(Y) d \sigma(X) \\
& +\int_{\partial \Omega_{h}} \tilde{a}(X) \int_{\partial \Omega} \nabla_{X} \Gamma_{X}^{t}(Y)\left(\nu(Y) \cdot A^{t}(Y) \nabla \varphi(Y)\right) \xi(Y-X) d \sigma(Y) d \sigma(X) .
\end{aligned}
$$

The first term here is a component of $\left\langle A_{0}, T^{t}\left(\eta B_{2}\right)\right\rangle$ and the second is again bounded by a constant, so we have from Calderón-Zygmund theory, Theorem 4.6 and Lemma 4.10 that

$$
\begin{aligned}
\left\|T^{t}\left(\eta B_{2}\right)\right\|_{\mathrm{BMO}} & \leq C+\left(C+C\left\|\widetilde{T}\left(B_{3}\right)\right\|_{\mathrm{BMO}}\right)\left\|B_{\varepsilon_{0}}\right\|_{L^{\infty}(\mathbf{R})} \\
& \leq C+C \varepsilon_{0}\left\|\widetilde{T}\left(B_{3}\right)\right\|_{\mathrm{BMO}},
\end{aligned}
$$

which leads to the second inequality.

It is now straightforward to complete the proof of Theorem 4.5 Lemma 4.11 allows us to conclude

$$
\left\|T^{t}\left(B_{2}\right)\right\|_{\text {вмо }} \leq C+C \varepsilon_{0}\left\|\widetilde{T}\left(B_{3}\right)\right\|_{\text {вмо }} \leq C+C \varepsilon_{0}\left(1+\left\|T^{t}\left(B_{2}\right)\right\|_{\text {вмо }}\right),
$$

so we can choose $\varepsilon_{0}$ sufficiently small, depending only on $k$ and the ellipticity constants, to hide the BMO-norm on the left-hand side and conclude

$$
\left\|T^{t}\left(B_{2}\right)\right\|_{\text {вмо }} \leq C \text {. }
$$

Note that we must check that our a priori assumptions imply that $\left\|T^{t}\left(B_{2}\right)\right\|_{\mathrm{BMO}}<$ $\infty$ in order to justify the last step. We can prove this quite quickly in our situation. First, observe that, via Theorem 4.6 and standard Calderón-Zygmund theory, it suffices to show $\left\|T^{t}\left(B_{4}\right)\right\|_{\text {BMO }}<\infty$ for some other bounded $B_{4}$ with bounded inverse. Also we have that $y \mapsto \phi(y)-\alpha_{0} y \in C_{0}^{\infty}(\mathbf{R})$ for some $\alpha_{0} \in \mathbf{R}$, so $\left\|\phi(y)-\alpha_{0} y\right\|_{L^{\infty}(\mathbf{R})}=M<\infty$. Choose $\eta \in C_{0}^{\infty}(\mathbf{R})$ so that $\eta(s)=1$ for $|s| \leq 2 M$. Setting $d_{0}(y, s)=s-\alpha_{0} y-\eta\left(s-\alpha_{0} y\right)\left(\phi(y)-\alpha_{0} y\right)$ and, applying Green's second identity to $\Gamma_{X}^{t}$ and $d_{0}$, we obtain

$$
d_{0}(X)-\int_{\Omega} \Gamma_{X}^{t} L^{t}\left(d_{0}\right)(Y) d Y=\int_{\partial \Omega} \Gamma_{X}^{t}(Y) \nu(Y) \cdot A^{t}(Y) \nabla d_{0}(Y) d \sigma(Y),
$$

since $d_{0} \equiv 0$ on $\partial \Omega$. Taking the gradient in $X$ we have

$$
\nabla d_{0}(X)-\int_{\Omega} \nabla_{X} \Gamma_{X}^{t} L^{t}\left(d_{0}\right)(Y) d Y=\int_{\partial \Omega} \nabla_{X} \Gamma_{X}^{t}(Y)\left(\nu(Y) \cdot A^{t}(Y) \nabla d_{0}(Y)\right) d \sigma(Y) .
$$

It is easy to check using (4.1) that both terms on the left are bounded, thus so is the right-hand side. But, if we choose $B_{4}: \mathbf{R} \rightarrow \mathcal{M}$ to be the diagonal matrix with diagonal entries both being $\nu \cdot A^{t} \nabla d_{0}$, then control of the right-hand side above 
controls the columns of $T^{t}\left(B_{4}\right)$, and $B_{4}$ is indeed bounded with a bounded inverse. Thus $\left\|T^{t}\left(B_{4}\right)\right\|_{L^{\infty}(\mathbf{R})}<\infty$ and so $\left\|T^{t}\left(B_{2}\right)\right\|_{\text {BMO }}<\infty$, as required.

We can then use (4.30) and Lemma 4.7 to apply Theorem 4.6 and obtain the desired bound on the $L^{2}$ operator norm of $T$ (and $\widetilde{T}$ via Lemmata 4.8, 4.10 and 4.11), completing the proof of Theorem 4.5,

\section{BOUNDEDNESS OF THE LAYER POTENTIALS ON BOUNDARIES WITH ARBITRARY LIPSCHITZ CONSTANTS}

The aim of this section is to remove the necessity for $\varepsilon_{0}$ in Theorem 4.5 to be small. We formulate this as the theorem below. It is proved by applying the build-up scheme of David [9] (see also [17]).

Theorem 5.1. The conclusion of Theorem 4.5 holds with $\alpha_{0}=0$ and $\varepsilon_{0}=k / 8$; that is, the conclusion holds for an arbitrary Lipschitz function $\phi$.

Theorem [5.1] will be proved using the following (9, Prop. 10] and [17, p. 110], respectively).

Theorem 5.2. Let $\varepsilon_{0}>0, \phi \in \Lambda^{k}\left(\varepsilon_{0}\right)$ be such that $\left\|\phi^{\prime}-\alpha_{0}\right\|_{L^{\infty}(\mathbf{R})} \leq \varepsilon_{0}$, and $I \subset \mathbf{R}$ be an interval. Then there exists a compact subset $E \subset I$ and a function $\psi \in \Lambda^{k+\frac{\varepsilon_{0}}{10}}\left(9 \varepsilon_{0} / 10\right)$ such that

$$
\begin{aligned}
& |E| \geq \frac{1}{3\left(1+\left(k+\varepsilon_{0}\right)^{2}\right)^{\frac{1}{2}}}|I|, \\
& \phi(x)=\psi(x) \text { for all } x \in E, \text { and either } \\
& -\frac{4}{5} \varepsilon_{0} \leq \psi^{\prime}(x)-\alpha_{0} \leq \varepsilon_{0} \text { or } \\
& -\varepsilon_{0} \leq \psi^{\prime}(x)-\alpha_{0} \leq \frac{4}{5} \varepsilon_{0} \text { almost everywhere. }
\end{aligned}
$$

Theorem 5.3. Let $K: \mathbf{R}^{2} \rightarrow \mathcal{M}$ be a Calderón-Zygmund kernel. Suppose that there exist constants $\theta \in(0,1]$ and $C_{9}>0$ such that the constant $C$ appearing in (2.1), (2.2) and (2.3) is no more than $C_{9}$ and, for all intervals $I$, there exists a compact subset $E \subset I$ and a Calderón-Zygmund kernel $K_{I}: \mathbf{R}^{2} \rightarrow \mathcal{M}$ having the following properties:

$$
\begin{aligned}
& |E| \geq \theta|I| \\
& \text { for all } x, y \in E \text { we have } K_{I}(x, y)=K(x, y) \text {; } \\
& \text { and }\left\|T_{I}^{*}\right\|_{L^{2}(\mathbf{R}) \rightarrow L^{2}(\mathbf{R})} \leq C_{9}
\end{aligned}
$$

where $T_{I}^{*}$ is the maximal singular integral operator associated to $K_{I}$. Then the maximal singular integral operator $T^{*}$ associated to $K$ is bounded on $L^{2}(\mathbf{R})$ with $\left\|T^{*}\right\|_{L^{2}(\mathbf{R}) \rightarrow L^{2}(\mathbf{R})} \leq C(\theta) C_{9}$.

Proof of Theorem [5.1. It suffices to show that Theorem 4.5 holds for $\phi \in \Lambda^{\frac{k}{8}}(k / 8)$. To this end, with $\varepsilon_{0}$ as in Theorem 4.5. pick $m$ so large that $(9 / 10)^{m} k / 8<\varepsilon_{0}$. Then Theorem 4.5 holds for $\phi \in \Lambda^{a_{m} k}\left((9 / 10)^{m} k / 8\right)$, where $a_{m}=1 / 4$.

We now claim Theorem 4.5 holds for $\phi \in \Lambda^{a_{m-1} k}\left((9 / 10)^{m-1} k / 8\right)$, where $a_{m-1}=$ $1 / 4-1 / 80(9 / 10)^{m-1}$. To see this fix $\phi \in \Lambda^{a_{m-1} k}\left((9 / 10)^{m-1} k / 8\right)$. Then for any interval $I$ apply Theorem 5.2 to obtain a compact subset $E \subset I$ and a Lipschitz function $\phi_{I} \in \Lambda^{a_{m} k}\left((9 / 10)^{m} k / 8\right)$. Denote by $K_{I}$, the kernel obtained via (4.7) 
as $K$ was but with $\phi$ replaced with $\phi_{I}$. Then $K_{I}$ satisfies the conditions of Theorem 5.3 with $\theta=1 /\left(3\left(1+k^{2}\right)^{\frac{1}{2}}\right)$. Indeed, Corollary 4.2 tells us that $K_{I}$ is a Calderón-Zygmund kernel, Theorem 4.5 tells us that $T_{I}^{*}$ is bounded on $L^{2}(\mathbf{R})$ and the remaining properties follow from Theorem 5.2 .

We can now repeat this argument to show that Theorem 4.5 holds for $\phi \in$ $\Lambda^{a_{m-2} k}\left((9 / 10)^{m-2} k / 8\right)$, where $a_{m-2}=1 / 4-(1 / 80)\left((9 / 10)^{m-1}+(9 / 10)^{m-2}\right)$. Continuing in this way, after $m$ steps we see that Theorem 4.5 holds for $\phi \in \Lambda^{a_{0} k}(k / 8)$, where

$$
a_{0}=\frac{1}{4}-\frac{1}{80} \sum_{j=0}^{m-1}\left(\frac{9}{10}\right)^{j}>\frac{1}{4}-\frac{1}{80} \sum_{j=0}^{\infty}\left(\frac{9}{10}\right)^{j}=\frac{1}{8} .
$$

Thus Theorem 5.1 is proved, and with it our main result, Theorem 1.4

\section{Appendix: An example}

Here we will show that given any $p>1$ there exist operators for which $(R)_{p}$ and $(N)_{p}$ do not hold.

Recall the operators $L_{h}=\operatorname{div} A \nabla \cdot$, for $h>0$, appearing in [19, Thm. (3.2.1)], where

and

$$
A=\left(\begin{array}{ll}
1 & m(x) \\
-m(x) & 1
\end{array}\right)
$$

$$
m(x)= \begin{cases}h, & x \geq 0 \\ -h, & x<0 .\end{cases}
$$

It is shown there that $u$ solves $L_{h} u=0$ in $\mathbf{R}_{+}^{2}$ if and only if $u$ is harmonic in the quarter planes $\{X=(x, t) \mid x>0, t>0\}$ and $\{X=(x, t) \mid x<0, t>0\}$, smooth up to the boundary except at $(0,0)$, continuous at $(0,0)$ and satisfying the transmission condition

$$
u_{x}^{-}-u_{x}^{+}-2 h u_{t}=0 \text { on }\{(x, t) \mid x=0\} .
$$

As a result they show that the function $w: \mathbf{R}_{+}^{2} \rightarrow \mathbf{R}$ given by

$$
w(x, t)= \begin{cases}\operatorname{Im}\left((x+i t)^{a}\right), & x \geq 0 \\ \operatorname{Im}\left((-x+i t)^{a}\right), & x<0\end{cases}
$$

satisfies $L_{h} w=0$ if and only if $h=\tan (b \pi / 2)$, where $b=1-a$. We can readily check that

$$
w=0, \partial_{x} w=0, \text { and } \partial_{t} w=a|x|^{-b} \text { on } \partial \mathbf{R}_{+}^{2} .
$$

Now solve (1.1) with data $f_{0} \in C_{0}^{\infty}\left(\partial \mathbf{R}_{+}^{2}, \mathbf{R}\right)$ such that $f_{0}=0$ for $|x|<1$ and $|x|>2, f_{0}=1$ for $9 / 8<|x|<15 / 8$, and $f_{0} \geq 0$ to obtain $u \in \widetilde{W}^{1,2}\left(\mathbf{R}_{+}^{2}\right)$ via Lemma 1.1. An application of the comparison principle [18, Lem. 1.3.7] shows that on $\{(x, t)|| x \mid<1 / 2, t=0\}$ we have

$$
\partial_{t} u \simeq \partial_{t} w
$$

thus $\partial_{t} u \simeq|x|^{-b}$ on the same set. Now, if $b p>1$, we have $\left\|\partial_{t} u\right\|_{L^{p}\left(\partial \mathbf{R}_{+}^{2}\right)}=$ $\infty$ and the regularity of the coefficients ensures that $\nabla u$ converges everywhere on the boundary except perhaps at $(0,0)$, so $\|\widetilde{N}(\nabla u)\|_{L^{p}\left(\partial \mathbf{R}_{+}^{2}\right)}=\infty$. However, $\left\|\partial_{x} u\right\|_{L^{p}\left(\partial \mathbf{R}_{+}^{2}\right)}<\infty$, so given any $p>1$ we can certainly find $b$ and $h$ so that $(R)_{p}$ does not hold for $L_{h}$ in $\mathbf{R}_{+}^{2}$. 
We can now show that $(N)_{p}$ cannot hold for the conjugate operator, which has coefficient matrix $\widetilde{A}=A^{t} / \operatorname{det}(A)$. Let $\widetilde{u}$ be the conjugate of $u$ defined by (2.10). Since $(u, \widetilde{u})$ satisfies (2.9),$\|\nabla u\|_{L^{2}(\Omega)}<\infty$ and again the regularity of the coefficients ensures that $\widetilde{u}$ is the unique solution to (1.2) with data $\nu \cdot \widetilde{A} \nabla \widetilde{u}$. Since the conormal derivative of $u$ becomes the tangential derivative of $\widetilde{u}$ and vice versa, $\|\nu \cdot \widetilde{A} \nabla \widetilde{u}\|_{L^{p}\left(\partial \mathbf{R}_{+}^{2}\right)}<\infty$ but $\left\|\partial_{x} \widetilde{u}\right\|_{L^{p}\left(\partial \mathbf{R}_{+}^{2}\right)}=\infty$; therefore, as before, we see $(N)_{p}$ cannot hold for this operator.

\section{ACKNOWLEDGEMENTS}

We would like to thank Steve Hofmann for helpful conversations and for making the related work by him and his coauthors [1] available to us.

\section{REFERENCES}

1. M. Alfonseca, P. Auscher, A. Axelson, S. Hofmann, and S. Kim, Analyticity of layer potentials and $L^{2}$ solvability of boundary value problems for divergence form elliptic equations with complex $L^{\infty}$ coefficients, to appear.

2. A.P. Calderón, Cauchy integrals on Lipschitz curves and related operators, Proc. Natl. Acad. Sci. 74 (1977), no. 4, 1324-1327. MR0466568 (57:6445)

3. R.R. Coifman, A. McIntosh, and Y. Meyer, L'integrale de Cauchy définit un opérateur borné sur $L^{2}$ pour les courbes lipschitziennes, Annals of Mathematics 116 (1982), 361-387. MR $672839(84 \mathrm{~m}: 42027)$

4. R.R. Coifman, Y. Meyer, and E.M. Stein, Some new function spaces and their applications to harmonic analysis, J. Funct. Anal. 62 (1985), no. 2, 304-335. MR791851 (86i:46029)

5. B.E.J. Dahlberg, Approximation of harmonic functions, Ann. Inst. Fourier 30 (1980), no. 2, 97-107. MR584274 (82i:31010)

6. - Weighted norm inequalities for the Lusin area integral and the nontangential maximal functions for functions harmonic in a Lipschitz domain, Studia Mathematica 67 (1980), 297314. MR592391 (82f:31003)

7. - Poisson semigroups and singular integrals, Proc. Amer. Math. Soc. 97 (1986), no. 1, 41-48. MR 831384 (87g:42035)

8. B.E.J. Dahlberg, C.E. Kenig, J. Pipher, and G.C. Verchota, Area integral estimates for higher order elliptic equations and systems, Ann. Inst. Fourier 47 (1997), no. 5, 1425-1461. MR.1600375 (98m:35045)

9. G. David, Opérateurs intégraux singuliers sur certaines courbes du plan complexe, Annales Scientifiques de l'É.N.S. 4 e série 17 (1984), no. 1, 157-189. MR744071 (85k:42026)

10. G. David, J.-L. Journé, and S. Semmes, Opérateurs de Calderón-Zygmund, fonctions paraaccrétives et interpolation, Rev. Mat. Iberoamericana 1 (1985), no. 4, 1-56. MR850408 (88f:47024)

11. L.C. Evans, Partial differential equations, American Mathematical Society, Providence, Rhode Island, 1999. MR 1625845 (99e:35001)

12. E.B. Fabes, M. Jodeit, Jr., and N.M. Rivière, Potential techniques for boundary value problems on $C^{1}$-domains, Acta Math. 141 (1978), 165-187. MR501367(80b:31006)

13. D. Gilbarg and N.S. Trudinger, Elliptic partial differential equations of second order, SpringerVerlag, Heidelberg, 1997.

14. L. Grafakos, Classical and modern Fourier analysis, Pearson Education Inc., Upper Saddle River, New Jersey, 2004.

15. T. Iwaniec and C. Sbordone, Riesz transforms and elliptic PDEs with VMO coefficients, J. Anal. Math. 74 (1998), 183-212. MR1631658 (99e:35034)

16. D.S. Jerison and C.E. Kenig, The Dirichlet problem in nonsmooth domains, Ann. of Math. 113 (1981), no. 2, 367-382. MR607897 (84j:35076)

17. J.-L. Journé, Calderón-Zygmund operators, pseudo-differential operators and the Cauchy integral of Calderón, Springer-Verlag, Heidelberg, 1983. 
18. C.E. Kenig, Harmonic analysis techniques for second order elliptic boundary value problems, CBMS Regional Conference Series, Amer. Math. Soc., Providence, Rhode Island, 1994. MR.1282720 (96a:35040)

19. C.E. Kenig, H. Koch, J. Pipher, and T. Toro, A new approach to absolute continuity of elliptic measure, with applications to non-symmetric equations, Advances in Mathematics 153 (2000), 231-298. MR.1770930 (2002f:35071)

20. C.E. Kenig and W.-M. Ni, On the elliptic equation $L u-k+K \exp [2 u]=0$, Ann. Scuola Norm. Sup. Pisa Cl. Sci. (4) 12 (1985), no. 2, 191-224. MR829052 (87f:35065)

21. C.E. Kenig and J. Pipher, The Neumann problem for elliptic equations with non-smooth coefficients, Invent. Math. 113 (1993), 447-509. MR1231834(95b:35046)

22. J. Pipher, Littlewood-Paley estimates: Some applications to elliptic boundary value problems, CRM Proceedings and Lecture Notes (Providence, Rhode Island), vol. 12, Amer. Math. Soc., 1997, pp. 221-238. MR 1479249 (98j:35047)

23. E.M. Stein, Singular integrals and differentiability properties of functions, Princeton University Press, Princeton, New Jersey, 1970. MR0290095 (44:7280)

24. - Harmonic analysis: Real-variable methods, orthogonality and oscillatory integrals, Princeton University Press, Princeton, New Jersey, 1993. MR.1232192 (95c:42002)

25. G.C. Verchota, Layer potentials and regularity for the Dirichlet problem for Laplace's equation in Lipschitz domains, Journal of Functional Analysis 59 (1984), 572-611. MR769382 (86e:35038)

26. G.C. Verchota and A.L. Vogel, Nonsymmetric systems on nonsmooth planar domains, Transactions of the American Mathematical Society 349 (1997), 4501-4535. MR 1443894 (98c:35040)

Department of Mathematics, University of Chicago, 5734 S. University Avenue, Chichgo, Illinois 60637

E-mail address: cek@math.uchicago.edu

Department of Mathematics, University of Chicago, 5734 S. University Avenue, Chicago, Illinois 60637

Current address: School of Mathematics, The University of Edinburgh, James Clerk Maxwell Building, The King's Buildings, Mayfield Road, Edinburgh, EH9 3JZ, United Kingdom

E-mail address: rule@uchicago.edu 